\title{
Estimación del riesgo ecológico y a la salud humana del mercurio en una zona de manglar del estuario La Puntilla, provincia de El Oro, sur del Ecuador
}

\section{Estimation of the ecological and human health risk of mercury in a mangrove area of the La Puntilla estuary, El Oro province, southern Ecuador}

\author{
Patricio Colón Velásquez-López ${ }^{1-2}$, Ivonne Yadira López Sánchez ${ }^{3}$ y María Fernanda Rivera Velásquez ${ }^{3-4}$ * \\ $\begin{array}{lll}\text { (D) } 0000-0002-3044-920 X & \text { (iD) } 0000-0002-8892-7913 & \text { (D) } 0000-0001-6453-2493\end{array}$ \\ 1. Universidad Técnica de Machala, Unidad de Ciencias Agropecuarias, Ecuador. \\ 2. Canadian International Resource and Development Institute, The University of British Columbia, Vancouver, Canadá. \\ 3. Escuela Superior Politécnica de Chimborazo, Grupo de investigación Ciencias de Datos, Facultad de Ciencias, Riobamba, Ecuador.maríaf.rivera@ \\ espoch.edu.ec *Autora para correspondencia. \\ 4. Universidad Nacional de Chimborazo, Facultad de Ingeniería, Riobamba, Ecuador.
}

\section{RESUMEN}

$\mathrm{E}$ 1 presente estudio se centra en la evaluación de los niveles de concentración de mercurio total y la estimación del riesgo ecológico (Er), el índice de riesgo ecológico (RI) y el coeficiente de riesgo tóxico o peligro (HQ) para la salud humana en un área de manglar junto a las desembocaduras de los ríos Chaguana y Siete, en el estuario de La Puntilla en el sur de Ecuador. Para la determinación de Er, RI y HQ, identificamos tres indicadores de contaminación por mercurio: sedimentos, raíz de mangle y tejido blando del bivalvo Anadara tuberculosa. En el área de manglar que bordea la desembocadura del río Chaguana, las concentraciones de mercurio fluctuaron entre $0,11 \pm 0,06 \mathrm{mg} / \mathrm{kg}$ en sedimentos, $0,06 \pm 0,01 \mathrm{mg} / \mathrm{kg}$ en raíz de mangle, con una consistente presencia de $A$. tuberculosa, pero solo una muestra del bivalvo registró un nivel de $0,034 \mathrm{mg} / \mathrm{kg}$ de mercurio. En contraste, en el área de manglar adyacente a la desembocadura del río Siete, las concentraciones de mercurio oscilaron entre $0,77 \pm 0,42 \mathrm{mg} / \mathrm{kg}$ en sedimentos y $0,15 \pm 0,12 \mathrm{mg} / \mathrm{kg}$ en la raíz de manglar, siendo imperceptible la presencia de A. tuberculosa. Los resultados del análisis de riesgo indicaron que en la desembocadura del río Chaguana, el Er y el índice de RI se colocaron en la categoría "baja". En contraste, en la desembocadura del río Siete, el Er resultó "alto" y RI resultó "moderado". El riesgo potencial para la salud humana fue bajo, en consistencia con el valor de $\mathrm{HQ}<1$ que considera el consumo de $A$. tuberculosa y el contacto dérmico a través de sedimentos; sin embargo, la escasa presencia del bivalvo en la desembocadura del río Siete es de preocupación.

PALABRAS CLAVE: índice de riesgo ecológico, mercurio, Anadara tuberculosa, ecosistema de manglar.

\section{ABSTRACT}

$\mathrm{P}$ resent study focused on the evaluation of total mercury concentration and the estimation of ecological risk (Er), the ecological risk index (IR); and the toxic risk or hazard quotient (HQ) for human health in a mangrove area that borders the mouths of the Chaguana and Siete rivers, in La Puntilla estuary in the south of Ecuador. For the determination of the Er, RI, and HQ, we identified three indicators of mercury contamination: sediments, mangrove root, and soft tissue of the bivalve mollusk Anadara tuberculosa. In the mangrove area that borders the mouth of the Chaguana river, the mercury concentration fluctuated between $0,11-0,06 \mathrm{mg} / \mathrm{kg}$ in sediments, $0.06-0,01 \mathrm{mg} / \mathrm{kg}$ in mangrove root, with a more consistence presence of $A$. tuberculosa, but one bivalve sample reported a level of $0,034 \mathrm{mg} / \mathrm{kg}$ of mercury. In contrast to the mangrove area adjacent to the mouth of the Siete river, whose concentrations ranged from 0,77-0,42 in sediments, and 0,15-0,12 in mangrove root, we found the highest mercury contamination, being imperceptible to the presence of A. tuberculosa. Results of the risk analysis indicated that, at the mouth of the Chaguana river, the Er and the RI index were placed in the "low" category. In contrast, at the mouth of the Siete river, the Er was "high," and RI was "moderate." Potential risk to human health was low, consistent with the value of $\mathrm{HQ}<1$ that considers the consumption of $A$. tuberculos $a$ and dermal contact through sediments; however, the low presence of the bivalve at the mouth of the Siete river is of concern.

KEYWORDS: ecological risk index, mercury, Anadara tuberculosa, mangrove ecosystem. 


\section{INTRODUCCIÓN}

Los procesos de contaminación generados por las actividades naturales y antrópicas en las cercanías de los cuerpos de agua son un problema cada vez más común que enfrentan los ecosistemas acuáticos. En regiones tropicales, la contaminación por metales pesados puede llegar a zonas de manglar, hábitat de especies marinas importantes para el sustento de comunidades costeras (Silva et al., 2003). Aproximadamente desde hace unos 25 años, mineros de la región sur del Ecuador utilizan mercurio para la recuperación del oro, considerándose una de las principales causas de contaminación en ríos y quebradas y superando en algunos casos los niveles permisibles establecidos según las normativas internacionales para agua de río y sedimentos (Velásquez-López et al., 2011). Se estima que en el 2010 la actividad minera de oro a nivel artesanal y en pequeña escala fue responsable del $29 \%$ de mercurio liberado a la atmósfera en América Latina y el Caribe (Santana et al., 2014). El desecho resultante de las actividades extractivas y de procesamiento mineral finalmente alcanza el cauce de ríos causando la acumulación de sedimentos enriquecidos con mercurio (Appleton et al., 2001; Carling et al., 2013). Por ejemplo, el mercurio desechado en las actividades mineras ha sido encontrado $250 \mathrm{~km}$ aguas abajo en el río Puyango al sur del Ecuador (Schudel et al., 2019). Una vez que el mercurio alcanza los ecosistemas acuáticos, puede precipitarse en el fondo (Marchand et al., 2006; Bazzi, 2014), permaneciendo allí durante largos períodos o bien, puede ser bioacumulado y biomagnificado en la cadena alimenticia (Morel et al., 1998; Chen et al., 2009; Le et al., 2017). Dentro de la cadena trófica, los moluscos bivalvos por su capacidad de filtración son reconocidos como organismos centinelas para biomonitoreo de mercurio en sistemas acuáticos (Maanan, 2008). En términos de riesgo a la salud humana, el consumo de moluscos bivalvos puede ser la principal vía de exposición al mercurio (World Health Organization, 1996); sin embargo, otra forma de exposición es el contacto dérmico a través de sedimentos contaminados (U.S. Environmental Protection Agency, 2001). Aceptando el peligro que representa la contaminación por mercurio, muchos de los países de la región han tomado medidas emergentes. Consecuentemente, en octubre del 2013, Ecuador suscribió su adhesión al Convenio de Minamata. Desde ese entonces, se ejecutan acciones para identificar puntos críticos de contaminación e incrementar las capacidades técnicas de monitoreo de mercurio en diferentes matrices ambientales para una aplicación eficaz de los compromisos adquiridos dentro del acuerdo internacional.

\section{INTRODUCTION}

Pollution processes generated by natural and anthropic activities in the vicinity of water bodies are an increasingly common problem facing aquatic ecosystems. In tropical regions, heavy metal contamination can reach mangrove areas, the habitat of important marine species for the sustenance of coastal communities (Silva et al., 2003). For approximately 25 years, miners in the southern region of Ecuador have used mercury for gold recovery, considering it one of the main causes of contamination in rivers and streams, in some cases exceeding the permissible levels established according to international regulations for river and sediments (Velásquez-López et al., 2011). It is estimated that in 2010 artisanal and small-scale gold mining activity was responsible for $29 \%$ of mercury released into the atmosphere in Latin America and the Caribbean (Santana et al., 2014). The waste resulting from extractive and mineral processing activities eventually reaches the riverbed causing the accumulation of mercury-enriched sediments (Appleton et al., 2001; Carling et al., 2013). For example, mercury released from mining activities was found $250 \mathrm{~km}$ downstream in the Puyango river in southern Ecuador (Schudel et al., 2019). Once mercury reaches aquatic ecosystems can precipitate to the bottom (Marchand et al., 2006; Bazzi, 2014), remaining for long periods, or can be bioaccumulated and biomagnified in the food chain (Morel et al., 1998; Chen et al., 2009; Le et al., 2017). Within the trophic chain, bivalve molluscs for their filtering capacity are recognized as sentinel organisms for biomonitoring of mercury in aquatic systems (Maanan, 2008). In terms of risk to human health, the consumption of bivalve molluscs may be the main route of exposure to mercury (World Health Organization, 1996); however, another form of exposure is dermal contact through contaminated sediments (US Environmental Protection Agency, 2001). Admitting the danger posed by mercury contamination, many of the countries in the region have taken emerging measures. Consequently, in October 2013, Ecuador signed its adhesion to the Minamata Agreement. Since then, actions are executed to identify critical points of contamination, thereby, increasing the technical capacities for mercury monitoring in different environmental matrices, for a useful application of the commitments acquired within the international agreement.

In southern Ecuador, $25 \mathrm{~km}$ away from the coastal zone for around 25 years, artisanal and smallscale miners use mercury for gold extraction. Previous 
En el sur del Ecuador, a $25 \mathrm{~km}$ de distancia de la zona costera, por alrededor de 25 años, mineros artesanales y pequeños mineros utilizan mercurio para la extracción de oro. Estudios previos sugieren que el mercurio puede alcanzar zonas de manglar, pero la información acerca de los niveles de concentración y posibles riesgos en estas áreas marítimas es limitada. Los manglares representan nichos ecológicos que albergan diversas especies de aves, peces y reptiles (Medina et al., 2007; Cardoso et al., 2009). A pesar de la importancia ecológica social y económica del ecosistema de manglar en el sur del Ecuador y la evidente contaminación por metales pesados en su proximidad, los riesgos asociados a la presencia de metales en estos ambientes frágiles han sido escasamente estudiados. En países en vías de desarrollo como Ecuador, los manglares benefician la práctica de la pesca artesanal y actividades de recreación, y debido a la potencial contaminación, existe interés en entender el estado ambiental de estas regiones para la formulación de medidas de protección y manejo. El objetivo del presente estudio fue determinar la concentración de mercurio en sedimentos, raíz de mangle y en el molusco bivalvo Anadara tuberculosa en dos sitios del estuario La Puntilla y estimar el Índice de Riesgo Ecológico (RI) y el Riesgo Tóxico o Hazard Quotient (HQ) para la salud humana. El escenario de investigación consideró el muestreo in situ en zonas de manglar del estuario colindantes a las desembocaduras de los ríos Chaguana y Siete para un análisis comparativo del 1) Índice de Riesgo Ecológico (RI), y 2) Riesgo Tóxico o Hazard Quotient (HQ) para la salud humana.

El análisis de riesgo sanitario ambiental se define como la cuantificación del daño toxicológico producido por efectos de un contaminante, que llega a un potencial receptor a través de diversas vías de migración y exposición (US. Environmental Protection Agency, 1989). Por otro lado, la estimación de riesgo ecológico es una herramienta utilizada para realizar diagnóstico cuantitativo de sensibilidad ambiental basado en el análisis de concentraciones de contaminantes (mercurio) en el sitio de estudio y su afectación al medio en que está presente (Hakanson, 1980). Aunque el presente estudio solo ofrece información esencial acerca de la contaminación y riesgos asociados al mercurio en una zona de manglar, es un significativo aporte al conocimiento sobre lo que, con seguridad, es un área de enorme interés académico respecto a la contaminación por metales pesados en zonas de manglar. El estudio beneficia a organismos internacionales studies have reported that mercury can reach mangrove areas, but the information about the concentratin levels and posible risks in these maritime áreas is limited. Mangroves represent ecological niches that embrace various species of birds, fish, and reptiles (Medina et al., 2007; Cardoso et al., 2009). Despite the ecological, social, and economic importance of the mangrove ecosystem in southern Ecuador and the evident contamination by heavy metals in its proximity, the risks associated with the presence of metals in these fragile environments have been scarcely studied. In developing countries such as Ecuador, mangroves benefit the practice of artisanal fishing and recreation activities, and due to potential polluting sources, there is interest in understanding the environmental state of these regions for the formulation of management and protection measures. The objective of the present study was to determine the concentration of mercury in sediments, mangrove root and in the bivalve mollusc Anadara tuberculosa at two sites in the La Puntilla estuary and to estimate the Ecological Risk Index (RI); and the Toxic Hazard or Hazard Quotient (HQ) for human health. The research scenario considered in situ samplings in mangrove areas of the estuary adjacent to the mouths of the Chaguana and Siete rivers for a comparative analysis of 1) Ecological Risk Index (RI), and 2) Toxic Risk or Hazard Quotient (HQ) for human health.

The environmental health risk analysis is known as the evaluation of toxicological damage caused by an effect of a contaminating that reach to a potential receptor through various migration and exposure pathways (US Environmental Protection Agency, 1989). On the other hand, an ecological risk estimation is a tool used to perform a quantitative diagnosis of environmental sensitivity based on the analysis of concentrations of pollutants (mercury) at the study site and its impact on the environment in which it is present (Hakanson, 1980). Although the current study only offers essential information about mercury contamination and risks in a mangrove area, it is a significant contribution to knowledge about what is undoubtedly an area of significant academic interest regarding heavy metal contamination in mangrove areas. The study benefits international organizations and organizations that work in ecological preservation and offers information to those in charge of formulating measures for the protection and management of coastal resources. 
y organizaciones que trabajan en la preservación ecológica, y ofrece información a aquellos que están encargados de formular medidas de protección y manejo de los recursos costeros.

\section{METODOLOGÍA}

El presente estudio se realizó en el bioma de manglar situado en las desembocaduras de los ríos Chaguana y Siete, en el estuario La Puntilla, provincia de El Oro, Ecuador. El área de estudio colinda con la provincia de Guayas hacia el norte y con la provincia de Azuay hacia el noroeste. El estuario de La Puntilla nace en la falda occidental de la Cordillera de Los Andes en los cantones Ponce-Enríquez (provincia del Azuay) y El Guabo, (provincia de El Oro), teniendo como principales afluentes a los ríos Chaguana y Siete. El flujo de agua en ambos ríos varía considerablemente. El río Siete tiene un promedio de $0,2-0,3 \mathrm{~m}^{3} / \mathrm{s}$ mientras que, en épocas de alta intensidad lluviosa, el caudal se incrementa a $2,5-7 \mathrm{~m}^{3} / \mathrm{s}$ (Secretaria Nacional de Planificación y Desarrollo, 2009). El río Chaguana está conectado con los ríos Pagua y Bonito, que corrientemente le aportan un caudal de alrededor de $4 \mathrm{~m}^{3} / \mathrm{s}$, constituyendo un cuerpo de agua significativamente más grande que el río Siete.

En los meses de febrero y agosto del año 2018 se realizaron cuatro campañas de muestreo en el ecosistema de manglar del Estuario la Puntilla junto a las desembocaduras de los ríos Chaguana y Siete (Figura 1). Se localizaron dos sitios de muestreo, uno junto a la desembocadura del río Chaguana, y otro junto a la desembocadura del río Siete. El estuario presenta dos pleamares y dos bajamares cada día. Los muestreos se realizaron en bajamar, cuando es posible acceder al terreno de manglar, ya que no está inundado por agua. Las muestras fueron tomadas en el fango del bioma de manglar donde habitan bivalvos y crustáceos. Para el muestreo se consideró indispensable el conocimiento de los pescadores artesanales, con quienes se planificó el acceso según el estado intermareal y acorde al desplazamiento de un conchero por jornada diaria (entre 4-6 horas). Con el acompañamiento de los pescadores artesanales se tomaron muestras de sedimentos, Anadara tuberculosa y raíz de mangle de la especie Rhizophora mangle sp. en cada sitio. Las muestras fueron tomadas de manera separada para evitar riesgos de contaminación cruzada. En cada estación se tomaron muestras simples de sedimentos y de raíz de mangle, acumuladas en recipientes separados e íntegramente limpios. Para el caso de A. tuberculosa, los

\section{METHODS}

The present study was conducted in the mangrove biome located at the mouths of the Chaguana and Siete rivers, in the La Puntilla estuary, El Oro province, Ecuador. The study area adjoins the Guayas province to the north and the Azuay province to the northwest. The La Puntilla estuary originates in the western foothills of the Andes Mountains in the Ponce-Enriquez (Azuay Province) and El Guabo (El Oro Province), having the Chaguana and Siete rivers as the main tributaries. The flow of water in both rivers varies considerably. The Siete river has an average of $0.2-0.3 \mathrm{~m}^{3} / \mathrm{s}$, while in times of high rain intensity, water flow increases to $2.5-7 \mathrm{~m}^{3} / \mathrm{s}$ (National Secretariat of Planning and Development, 2009). The Chaguana river is connected to the Pagua and Bonito rivers, which currently provide a flow of around $4 \mathrm{~m}^{3} / \mathrm{s}$, constituting a more significant body of water than the Siete river.

In February and August of 2018, four sampling campaigns were carried out in the mangrove ecosystem of the La Puntilla estuary next to the mouths of the Chaguana and Siete rivers (Figure 1). Two sampling sites were located, one next to the mouth of the Chaguana river, and another next to the mouth of the Siete river. The estuary daily features two high tides and two low tides. The samplings were carried out at low tide when it is possible to access the mangrove land since it is not flooded by water. The samples were taken in the mangrove biome mudflat, habitat of bivalves, and crustaceans. For the sampling, it was essential to consider local artisanal fishers' knowledge with whom access was planned according to the intertidal state and in the function of the movement of one fisherman per day (between 4-6 hours) during his daily journey. With the accompaniment of artisanal fishers, we sampled the Anadara tuberculosa and obtained the mangrove root of the Rhizophora mangle sp species at each site. The samples were taken separately to avoid risks of cross-contamination. Sediment and mangrove root samples were collected at each station, accumulated in separate and clean containers. In the case of $A$. tuberculosa, the organisms were placed in baskets after superficial cleaning, ruling out the presence of sediment and mud in the external valves. On each sampling day, the materials and utensils were washed and rinsed with $5 \%$ nitric acid. 


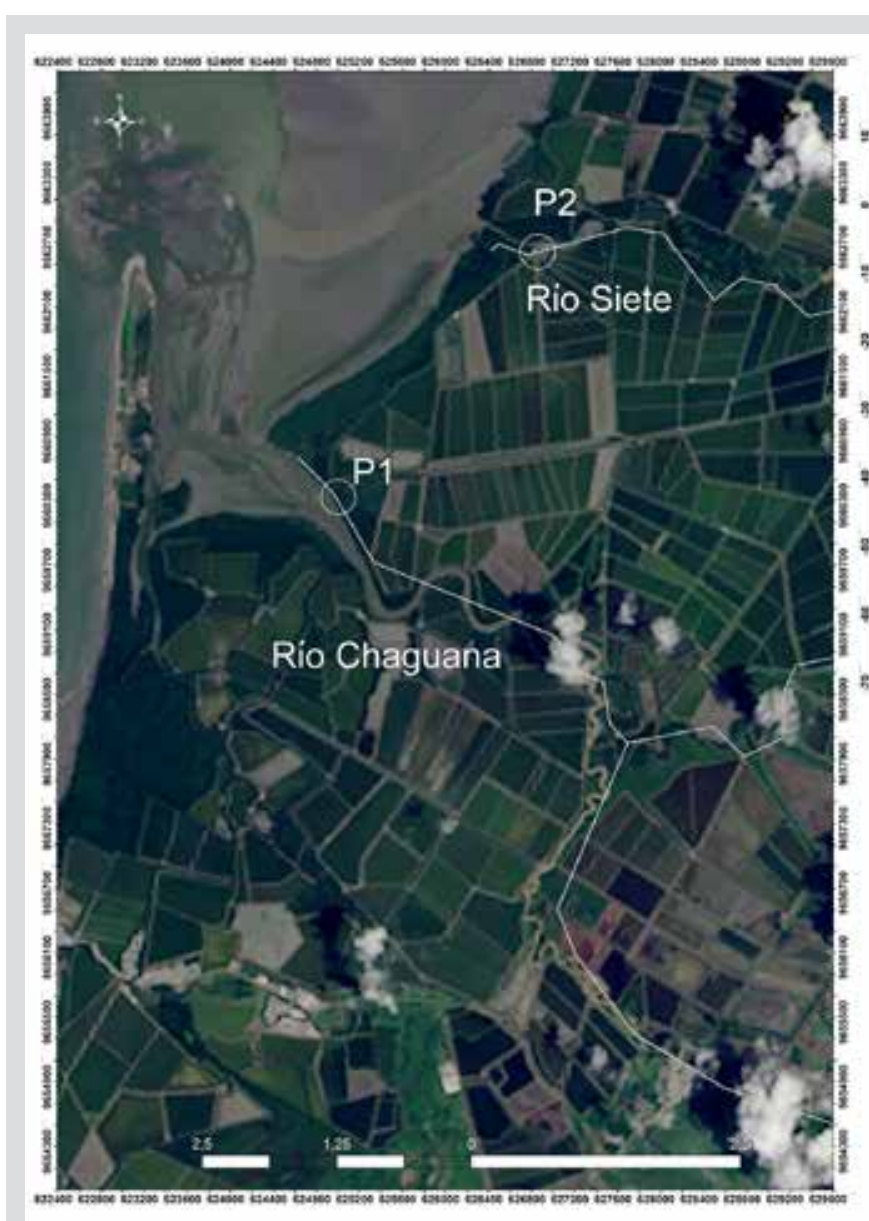

Figura 1. Zona de estudio: Estuario La Puntilla, perteneciente a Bajo Alto, cantón El Guabo, provincia de El Oro, Ecuador.

organismos fueron colocados en canastillas luego de una limpieza superficial para desechar la presencia de sedimento y lodo en las valvas externas. En cada jornada de muestreo, los materiales y utensilios se lavaron y enjuagaron con ácido nítrico al $5 \%$.

\section{Muestreo de sedimentos}

En cada punto, siguiendo la secuencia del pescador artesanal y usando un tubo PVC de 6 pulgadas de diámetro, se tomaron al azar aproximadamente $3000 \mathrm{~g}$ de sedimento en varios puntos a una profundidad de alrededor $20 \mathrm{~cm}$. De las muestras simples de sedimentos se constituyó una muestra compuesta de alrededor de $500 \mathrm{~g}$, la cual fue empaquetada en fundas plásticas con cierre hermético y almacenada en un recipiente especialmente preparado para los sedimentos. Posteriormente, las muestras fueron sometidas a secado, molienda y tamizado $(75 \mu \mathrm{m}, 150 \mu \mathrm{m}$, $300 \mu \mathrm{m}$ y $850 \mu \mathrm{m})$ y empaquetadas herméticamente para su posterior transporte al laboratorio analítico.

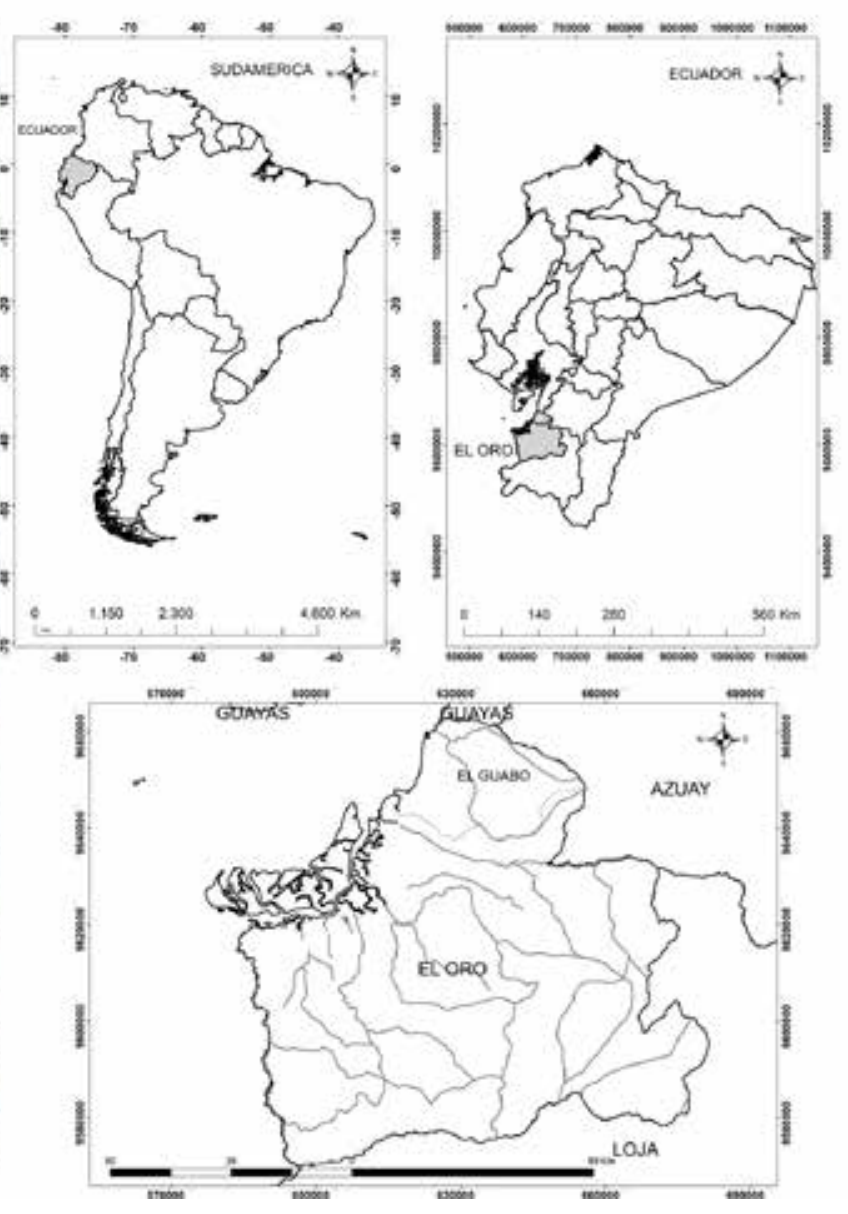

Figure 1. Study area: La Puntilla estuary, belonging to Bajo Alto, El Guabo canton, El Oro province, Ecuador.

\section{Sediment sampling}

At each point following the artisanal fisherman's sequence and using a 6-inch diameter PVC tube, approximately $3000 \mathrm{~g}$ of sediment were randomly taken at various locations at a depth of about 20 centimeters. A composite sample of around $500 \mathrm{~g}$ was formed from the discrete sediment samples, which was packed in plastic covers with hermetic closure and placed in a container specially prepared for the sediments. Subsequently, the samples were subjected to drying, grinding, and sieving (75 $\mu \mathrm{m}, 150 \mu \mathrm{m}, 300 \mu \mathrm{m}$, and $850 \mu \mathrm{m})$ and hermetically packed for subsequent transport to the analytical laboratory.

\section{Root sampling of Rhizophora sp.}

At the two sampling sites previously described, and following the trajectory of the artisanal fisherman, samples of the mangrove root were obtained. Mangrove root samples were taken by cutting off the soft tissue at 
Muestreo de raíz de Rhizophora sp.

En los dos sitios de muestreo previamente descritos y siguiendo la trayectoria del pescador artesanal, se obtuvieron muestras de la raíz de manglar, las cuales fueron tomadas cortando la parte final y blanda de la raíz suspendida. Por lo general, la raíz de mangle contiene partículas en suspensión y microorganismos. Las muestras con todo su contenido fueron almacenadas. Una muestra consistió en varios fragmentos de raíz de mangle para completar aproximadamente $250 \mathrm{~g}$. La muestra fue colocada en fundas plásticas con cierre hermético y dispuesta en un recipiente en frío.

\section{Muestreo de Anadara tuberculosa}

La captura de bivalvos se realizó con especial atención a la obtención de la concha negra A. tuberculosa, la cual se realizó con ayuda del pescador quien fue el único que extrajo el bivalvo del fango del hábitat de manglar a una profundidad alrededor de $20 \mathrm{~cm}$. Las conchas fueron recibidas por otra persona con manos limpias que se dedicó al manejo de estas, esto es, al enjuague en agua de mar para eliminar el exceso de sedimentos, empaquetado y almacenamiento en el recipiente colector. En laboratorio y bajo íntegras condiciones de asepsia, se realizó la disección del organismo bivalvo para extraer el tejido blando asegurando un peso de alrededor de $100 \mathrm{~g}$ acorde a lo requerido por el laboratorio analítico. Del número de individuos capturados en las cuatro campañas de muestreo, se obtuvieron 22 muestras que fueron analizadas. Cada muestra consistió en alrededor de 20 a 30 ejemplares de A. tuberculosa. Se registró tamaño y peso de los individuos capturados medidos por medio de un escalímetro y una balanza digital con sensibilidad a $0,1 \mathrm{~g}$, respectivamente. El almacenamiento de muestras de sedimentos, raíz de mangle y A. tuberculosa fue realizado por separado con recipientes térmicos para preservación a bajas temperaturas y teniendo presente criterios de conservación y transporte de muestras (Instituto de Investigaciones Marinas y Costeras, 2013).

\section{Análisis de mercurio}

Las concentraciones de mercurio total en las muestras de sedimento, raíz de manglar y del bivalvo A. tuberculosa fueron analizadas en el laboratorio de la Subsecretaría de Calidad e Inocuidad del Ministerio de Acuacultura y Pesca del Ecuador, con acreditación para la determinación de mercurio. El método analítico consistió en la técnica de vapor frío con el uso de un espectrofotómetro de absorción atómica (método de referencia P1-MP1 VARIAN AA 60), de acuerdo con procedimientos intrínsecos para el the suspended plant root. In general, the mangrove root contains suspended particles and microorganisms. The root samples were stored with all their content. A sample consisted of several mangrove root fragments to complete approximately $250 \mathrm{~g}$, which was placed in plastic bags with a hermetic seal and placed in a cold container.

\section{Sampling of Anadara tuberculosa}

The capture of bivalves was carried out with particular attention to obtaining the black shellfish $A$. tuberculosa, which was carried out with the help of the fisherman, who was the only one who extracted the bivalve from the mud of the mangrove habitat at a depth of around $20 \mathrm{~cm}$. Another person received the shellfishes with clean hands for handling rinsing them in seawater to remove excess sediment, packaging, and storage in the collecting container. In the laboratory and under thorough aseptic conditions, the bivalves were dissected, extracting the soft tissue. A sample weight of around $100 \mathrm{~g}$ was required for analytical purposes at the laboratory. From the total number of individuals captured in the four sampling campaigns, 22 samples were obtained and analyzed. Each sample consisted of between 20 to 30 specimens of A. tuberculosa. The size and weight of the captured individuals were recorded, measured utilizing a scale and a digital scale with sensitivity to $0.1 \mathrm{~g}$, respectively. The storage of samples of sediments, mangrove root and A. tuberculosa was carried out separately, using thermal containers for preservation at low temperatures and keeping sample conservation and transport criteria in mind (Instituto de Investigaciones Marinas y Costeras, 2013).

\section{Mercury analysis}

The concentrations of total mercury in the samples of sediment, mangrove root and the bivalve A. tuberculosa were analyzed in the laboratory of the Undersecretary of Quality and Safety, of the Ministry of Aquaculture and Fisheries of Ecuador with accreditation for the determination of mercury in biological samples. The analysis was performed by using the cold vapor technique using the Atomic Absorption Spectrophotometer (reference method P1-MP1 VARIAN AA 60), and according to intrinsic analytical procedures of the laboratory. The detection limit reported by the laboratory was $0.09 \mathrm{mg} / \mathrm{kg} \mathrm{Hg}$, and the reported values correspond to the dry weight of the samples. The mercury concentration results were analyzed using a one-way analysis of variance (ANOVA) to determine if there is a significant difference 
análisis en el laboratorio. El límite de detección reportado por el laboratorio fue de $0,09 \mathrm{mg} / \mathrm{kg}$ de $\mathrm{Hg}$ y los valores reportados corresponden al peso seco de las muestras. Los resultados de la concentración de mercurio fueron analizados mediante análisis de varianza de una vía (ANOVA) para determinar si existe diferencia significativa entre los promedios determinados para cada componente en los dos puntos del estero La Puntilla.

\section{Cálculo del riesgo ecológico}

Para el cálculo del riesgo ecológico, se utilizó el método descrito por Hakanson (1980), que tiene como propósito identificar la concentración de metales pesados en la muestra para evaluar el factor de contaminación $\left(\mathrm{C}_{\mathrm{f}}^{\mathrm{i}}\right)$, el potencial de riesgo ecológico ( $\left.\mathrm{Er}^{\mathrm{i}}\right)$ y el índice de potencial riesgo ecológico (RI). El factor de contaminación identifica qué tan contaminante puede llegar a ser el mercurio y se calculó de la siguiente manera:

$$
\mathrm{C}_{\mathrm{f}}^{\mathrm{i}}=\mathrm{C}_{0-1}^{\mathrm{i}} / \mathrm{C}_{\mathrm{n}}^{\mathrm{i}} \quad \text { Ec. } 1
$$

Donde,

$\mathrm{C}_{0-1}^{\mathrm{i}}$ es la concentración de mercurio en la muestra

$\mathrm{C}_{\mathrm{n}}^{\mathrm{i}}$ corresponde a los valores de referencia de mercurio cuyo valor propuesto por Hakanson es de 0,25

El factor potencial de riesgo ecológico ayuda a conocer el riesgo ambiental ( $\left.\mathrm{Er}^{\mathrm{i}}\right)$ que puede tener el mercurio con base en la relación del factor de contaminación y los valores de respuesta tóxica, y se interpretan de la siguiente manera:

$$
\operatorname{Er}^{\mathrm{i}}=\operatorname{Tr}^{\mathrm{i} *} \mathrm{C}_{\mathrm{f}}^{\mathrm{i}} \quad \text { Ec. } 2
$$

Donde,

$\operatorname{Tr}^{\mathrm{i}}$ corresponde al factor de respuesta tóxica de la sustancia, con un valor de 40 para el mercurio

$\mathrm{C}_{\mathrm{f}}^{\mathrm{i}}$ representa al factor de contaminación

El índice de riesgo ecológico (RI) integra los factores de potencial de riesgo ecológico del mercurio y permite establecer si las concentraciones en la muestra de estudio tienen riesgo ambiental. La categoría del RI varía desde bajo a alto y se calculó con la siguiente ecuación:

$$
\mathrm{RI}=\sum \operatorname{Er}^{\mathrm{i}} \text { Ec. } 3
$$

La interpretación del factor de contaminación potencial de riesgo ecológico e índice de riesgo ecológico se resume en la Tabla 1, donde se presentan los valores y sus respectivas categorías. between the averages determined for each component in the two points of the La Puntilla estuary.

\section{Ecological risk calculation}

To calculate the ecological risk, we used the method described by Hakanson (1980), which aims to identify the concentration of heavy metals on the sample to assess the pollution factor $\left(\mathrm{C}_{\mathrm{f}}^{\mathrm{i}}\right)$, ecological risk potential $\left(\mathrm{Er}^{\mathrm{i}}\right)$, ecological risk potential index (RI). The pollution factor identifies how polluting, mercury can be in the study site, and was calculated as follows:

$$
\mathrm{C}_{\mathrm{f}}^{\mathrm{i}}=\mathrm{C}_{0-1}^{\mathrm{i}} / \mathrm{C}_{\mathrm{n}}^{\mathrm{i}} \quad \text { Eq. } 1
$$

Where,

$\mathrm{C}_{0-1}^{\mathrm{i}}$ is the concentration of mercury in the sample

$\mathrm{C}_{\mathrm{n}}^{\mathrm{i}}$ corresponds to the reference mercury values whose value proposed by Hakanson is 0.25

The potential ecological risk factor helps to know the ecological risk $\left(E^{i}\right)$ that mercury can have based on the relationship between the pollution factor and the toxic response values. They are interpreted as follows:

$$
\operatorname{Er}^{\mathrm{i}}=\operatorname{Tr}^{\mathrm{i} *} \mathrm{C}_{\mathrm{f}}^{\mathrm{i}} \quad \text { Eq. } 2
$$

Where,

$\operatorname{Tr}^{\mathrm{i}}$ corresponds to the toxic response factor of the substance, with a value of 40 for mercury

$\mathrm{C}_{\mathrm{f}}^{\mathrm{i}}$ represents the pollution factor

The ecological risk index (RI) integrates the ecological risk potential factors of mercury and allows establishing whether the concentrations in the study sample have ecological risk. The RI category was calculated with the following equation.

$$
\mathrm{RI}=\sum \mathrm{Er}^{\mathrm{i}} \quad \text { Eq. } 3
$$

The interpretation of the potential ecological risk pollution factor and ecological risk index is summarized in Table 1, where the values and their respective categories are presented. 
Tabla 1. Categorías de interpretación del factor de contaminación potencial de riesgo ecológico e índice de riesgo ecológico.
Table 1. Interpretation categories of the potential ecological risk pollution factor and ecological risk index.

\begin{tabular}{|c|c|c|c|c|c|}
\hline \multicolumn{2}{|c|}{ Factor de contaminación/Pollution factor } & \multicolumn{2}{|c|}{$\begin{array}{l}\text { Potencial de riesgo ecológico/ } \\
\text { Ecological risk potential }\end{array}$} & \multicolumn{2}{|c|}{ Índice de riesgo/Risk index } \\
\hline Valor/Value & Categoría/Category & Valor/Value & Categoría/Category & Valor/Value & Categoría/Category \\
\hline $\mathrm{C}_{\mathrm{f}}^{\mathrm{i}}<1$ & Bajo/Low & $\operatorname{Er}^{\mathrm{i}}<40$ & Bajo/Low & $\mathrm{RI} \leq 150$ & Bajo/Low \\
\hline $1 \leq \mathrm{C}_{\mathrm{f}}^{\mathrm{i}}<3$ & Moderado/Moderate & $40 \leq \operatorname{Er}^{\mathrm{i}}<80$ & Moderado/Moderate & $150 \leq \mathrm{RI}<300$ & Moderado/Moderate \\
\hline $3 \leq \mathrm{C}_{\mathrm{f}}^{\mathrm{i}}<6$ & Considerable & $80 \leq \operatorname{Er}^{\mathrm{i}}<160$ & Considerable & $300 \leq \mathrm{RI}<600$ & Considerable \\
\hline \multirow[t]{2}{*}{$6 \leq \mathrm{C}_{\mathrm{f}}^{\mathrm{i}}$} & Muy alto/Very high & $160 \leq \operatorname{Er}^{\mathrm{i}}<320$ & Muy alto/Very high & $\mathrm{RI} \geq 600$ & Muy alto/Very high \\
\hline & & $\operatorname{Er}^{\mathrm{i}} \geq 320$ & $\begin{array}{l}\text { Muy alto riesgo } \\
\text { ecológico/Very high } \\
\text { ecological risk }\end{array}$ & & \\
\hline
\end{tabular}

\section{Análisis de riesgo sanitario ambiental}

El cálculo del riesgo sanitario ambiental se realizó tomando en cuenta vías activas de exposición: ingestión (Ec. 4) y contacto dérmico (Ec. 5). Se considera que el sitio de estudio representa un hábitat donde se asientan pequeños grupos familiares que aprovechan el manglar para la pesca artesanal y, por tanto, subsisten de este recurso. Por otro lado, se calculó el riesgo producido por la ingesta de mercurio a través del consumo de conchas del género A. tuberculosa (Ec. 6), considerando que este bivalvo es distribuido y consumido en diversas partes de la región. La Tabla 2 muestra la descripción de las variables, los valores y las unidades utilizadas.

\section{$\mathrm{C}_{\mathrm{sn}} * \mathrm{CF}_{1} * \mathrm{EF}^{*} \mathrm{FI}^{*} \mathrm{ED} * \mathrm{IR}$ $\mathrm{CF}_{2} * \mathrm{BW}^{*} \mathrm{AT} * \mathrm{RfDs}$

$$
\frac{\mathrm{C}_{\mathrm{sn}}{ }^{*} \mathrm{CF}_{3} * \mathrm{SA}^{*} \mathrm{AF}^{*} \mathrm{ABS} * \mathrm{EF} * \mathrm{ED}}{\mathrm{CF}_{2}{ }^{*} \mathrm{BW}^{*} \mathrm{AT} * \mathrm{RfDs}}
$$

$\mathrm{Ct} * \mathrm{EF}^{*} \mathrm{ED} * \mathrm{FIR}^{*} \mathrm{CF}_{4}$ $\mathrm{RfDt}^{*} \mathrm{BW} * \mathrm{AT}$

Ec. 4

Ec. 5

Ec. 6

\section{Environmental health risk analysis}

The environmental health risk calculation was carried out, taking into consideration active exposure routes such as ingestion (Eq. 4) and Dermal Contact (Eq. 5). We consider that the study site is a habitat where small family groups settle that take advantage of the mangrove for artisanal fishing by both subsist on this resource. On the other hand, the risk produced by the ingestion of mercury through the consumption of shells of the genus $A$. tuberculosa (Eq. 6) was calculated considering that this bivalve is distributed and consumed in various parts of the region. Table 2 shows a description of the variables, values, and units used.

$\mathrm{C}_{\mathrm{sn}} * \mathrm{CF}_{1} * \mathrm{EF}^{*} \mathrm{FI}^{*} \mathrm{ED}^{*} \mathrm{IR}$

Eq. 4 $\mathrm{CF}_{2}{ }^{*} \mathrm{BW}^{*} \mathrm{AT} * \mathrm{RfDs}$

$\mathrm{C}_{\mathrm{sn}} * \mathrm{CF}_{3} * \mathrm{SA}^{*} \mathrm{AF}^{*} \mathrm{ABS} * \mathrm{EF} * \mathrm{ED}$ $\mathrm{CF}_{2} * \mathrm{BW}^{*} \mathrm{AT} * \mathrm{RfDs}$

Eq. 5
Eq. 6
$\underline{\mathrm{Ct} * \mathrm{EF}^{*} \mathrm{ED}^{*} \mathrm{FIR}^{*} \mathrm{CF}_{4}}$ $\mathrm{RfDt}^{*} \mathrm{BW} * \mathrm{AT}$ 
Tabla 2. Valores empleados para el cálculo del riesgo.

Table 2. Values used to calculate risk.

\begin{tabular}{|c|c|c|c|c|}
\hline \multicolumn{3}{|c|}{ Ingestión incidental por sedimentos/Incidental sediment ingestión } & \multicolumn{2}{|c|}{ Receptores/Receivers } \\
\hline \multicolumn{2}{|l|}{ Factores/Factors } & Unidades/Units & \multirow{2}{*}{$\begin{array}{l}\text { Adultos/Adults } \\
\text { Concentración - metal/ } \\
\text { Concentration - metal }\end{array}$} & \multirow{2}{*}{$\begin{array}{c}\text { Niños/Children } \\
\text { Concentración - metal/ } \\
\text { Concentration - metal }\end{array}$} \\
\hline Concentración del elemento/Element concentration & Csn & $\mathrm{mg} / \mathrm{kg}$ & & \\
\hline Factor de conversión 1/Conversion factor 1 & CF1 & $\mathrm{kg} / \mathrm{mg}$ & $1 \times 10^{-5}$ & $1 \times 10^{-5}$ \\
\hline Frecuencia de exposición/Exposure frequency & $\mathrm{EF}$ & $\begin{array}{l}\text { Día/año } \\
\text { Day/year }\end{array}$ & 350 & 350 \\
\hline Fracción de ingestión/Ingestion fraction & FI & $\begin{array}{l}\text { Adimensional/ } \\
\text { Dimensionless }\end{array}$ & 1 & 1 \\
\hline Duración de exposición/Exposure duration & ED & Año/Year & 24 & 6 \\
\hline Tasa de ingestión del suelo/Soil ingestion rate & IR & $\mathrm{mg} /$ día & 100 & 200 \\
\hline Factor de conversión 2/Conversion factor 2 & $\mathrm{CF}_{2}$ & $\begin{array}{l}\text { día/año } \\
\text { Day/year }\end{array}$ & 365 & 365 \\
\hline Peso corporal/Body weight & BW & $\mathrm{kg}$ & 70 & 15 \\
\hline Tiempo promedio/Average time & AT & Año/Year & $\begin{array}{l}\text { LT (cancerígeno/ } \\
\text { carcinogenic) } \\
\text { ED (no cancerígeno/ } \\
\text { non-carcinogenic) }\end{array}$ & $\begin{array}{l}\text { LT (cancerígeno/ } \\
\text { carcinogenic) } \\
\text { ED (no cancerígeno/ } \\
\text { non-carcinogenic) }\end{array}$ \\
\hline Dosis de referencia Sedimentos/Sediment reference dose & RfDs & $\begin{array}{l}\mathrm{mg} / \mathrm{kg} * \text { día } \\
\mathrm{mg} / \mathrm{kg} \text { *day }\end{array}$ & $8.57 \times 10^{-04}$ & $8.57 \times 10^{-04}$ \\
\hline \multicolumn{3}{|c|}{ Contacto dérmico incidental por sedimentos/Incidental dermal contact by sediment } & \multicolumn{2}{|c|}{ Receptores/Receivers } \\
\hline \multicolumn{2}{|l|}{ Factores/Factors } & Unidades/Units & Adultos/Adults & Niños/Children \\
\hline Factor de conversión 3/Conversion factor 3 & CF3 & $\mathrm{kg} * \mathrm{~cm}^{2} / \mathrm{mg}^{*} \mathrm{~m}^{2}$ & 0.01 & 0.01 \\
\hline Área de superficie/Surface area & SA & $\begin{array}{l}\mathrm{m}^{2} / \text { día } \\
\mathrm{m}^{2} / \text { day }\end{array}$ & 0.53 & 0.53 \\
\hline Factor de adherencia/Adhesion factor & $\mathrm{AF}$ & $\mathrm{mg} / \mathrm{cm}^{2}$ & 1 & 1 \\
\hline Factor de absorción/Absorption factor & ABS & $\begin{array}{l}\text { Adimensional/ } \\
\text { Dimensionless }\end{array}$ & $\begin{array}{c}\text { 0.01(orgánico/organic) } \\
0.001 \text { (inorgánico/ } \\
\text { inorganic) }\end{array}$ & $\begin{array}{c}\text { 0.01(orgánico/organic) } \\
0.001 \text { (inorgánico/ } \\
\text { inorganic) }\end{array}$ \\
\hline Duración de exposición/Duration of exposition & ED & Año/Year & 30 & 30 \\
\hline Factor de conversión 2/Conversion factor 2 & $\mathrm{CF}_{2}$ & $\begin{array}{l}\text { Día/año } \\
\text { Day/year }\end{array}$ & 365 & 365 \\
\hline Dosis de referencia Sedimentos/Sediment reference dose & RfDs & $\begin{array}{l}\mathrm{mg} / \mathrm{kg}^{*} \text { día } \\
\mathrm{mg} / \mathrm{kg}^{*} \text { day }\end{array}$ & $3 \times 10^{-05}$ & $3 \times 10^{-05}$ \\
\hline \multicolumn{3}{|c|}{ Ingestión de Anadara tuberculosa/Ingestion of Anadara tuberculosa } & \multicolumn{2}{|c|}{ Receptores/Receivers } \\
\hline \multicolumn{2}{|l|}{ Factores/Factors } & Unidades/Units & Adultos/Adults & Niños/Children \\
\hline Duración de exposición/Exposure duration & ED & Año/Year & 70 & 6 \\
\hline Tasa de ingestión de alimento/Food ingestion rate & FIR & $\begin{array}{l}\text { gr/persona/día } \\
\text { gr/person/day }\end{array}$ & 9.8 & 9.8 \\
\hline Factor de conversión 4/Conversion factor 4 & $\mathrm{CF}_{4}$ & $\mathrm{~kg} / \mathrm{g}$ & 0.001 & 0.001 \\
\hline Dosis de referencia tejido blando/Soft tissue reference dose & RfDt & $\begin{array}{l}\text { mg/kg-día } \\
\text { mg/kg-day }\end{array}$ & 0.003 & 0.003 \\
\hline Peso corporal/Body weight & BW & $\mathrm{kg}$ & 70 & 15 \\
\hline Tiempo promedio/Average time & AT & Año/Year & $\mathrm{EF}^{*} \mathrm{ED}$ & $\mathrm{EF}^{*} \mathrm{ED}$ \\
\hline
\end{tabular}

El $\mathrm{HQ}$ es un índice de peligro que identifica cuantitativamente si la exposición al mercurio supera la dosis tolerable o de referencia RfD (Reference dose) $[\mathrm{mg} / \mathrm{kg} / \mathrm{d}$ ] La RfD estima la exposición media diaria que no produce efectos adversos apreciables en el organismo humano
The HQ is a hazard index that quantitatively identifies whether exposure to mercury exceeds the tolerable or reference dose RfD (reference dose) [mg/kg d]. The RfD estimates the average daily exposure that does not produce measurable adverse effects on the human organism during 
durante el trascurso de su vida. El coeficiente de peligro total se considera como la suma de los coeficientes individuales. Para la determinación del HQ total se realizó la siguiente sumatoria donde se considera a vía de exposición i-ésima.

$$
\mathrm{HQ}_{\mathrm{tot}}=\sum_{1}^{\mathrm{n}} \mathrm{HQ}_{\mathrm{i}} \quad \text { Ec. } 7
$$

Los estándares para la protección humana definidos por diversos organismos internacionales preveen los siguientes criterios de aceptabilidad: para el riesgo no cancerígeno (exposición a una o más sustancias) HQ, HQ $<1.0$ (Environmental Protection Agency, 1997).

\section{RESULTADOS}

Concentración de mercurio en sedimentos, raíz de mangle y bivalvo Anadara tuberculosa

En la Figura 2 se muestran los resultados de los niveles de concentración de mercurio $(\mathrm{Hg})$ en sedimentos, tejido blando de Anadara tuberculosa y raíz de mangle detectados en el estuario La Puntilla a lo largo del lecho de manglar que colinda con las desembocaduras de los ríos Siete y Chaguana. its life. The total hazard coefficient is considered as the sum of the individual factors. For the determination of the total $\mathrm{HQ}$, the following summation was performed, taking into account the route of exposure.

$$
\mathrm{HQ}_{\mathrm{tot}}=\sum_{1}^{\mathrm{n}} \mathrm{HQ}_{\mathrm{i}}
$$

The standards for personal protection defined by various international organizations provide the following acceptability criteria: for non-carcinogenic risk (exposure to one or more substances) HQ, HQ $<1.0$ (Environmental Protection Agency, 1997).

\section{RESULTS}

Mercury concentration in sediments, mangrove root, and bivalve Anadara tuberculosa

Figure 2 shows the results of mercury $(\mathrm{Hg})$ concentration levels in sediments, the soft tissue of Anadara tuberculosa and mangrove root detected in the La Puntilla estuary along the mangrove bed that adjoins the mouths of the Siete and Chaguana rivers.

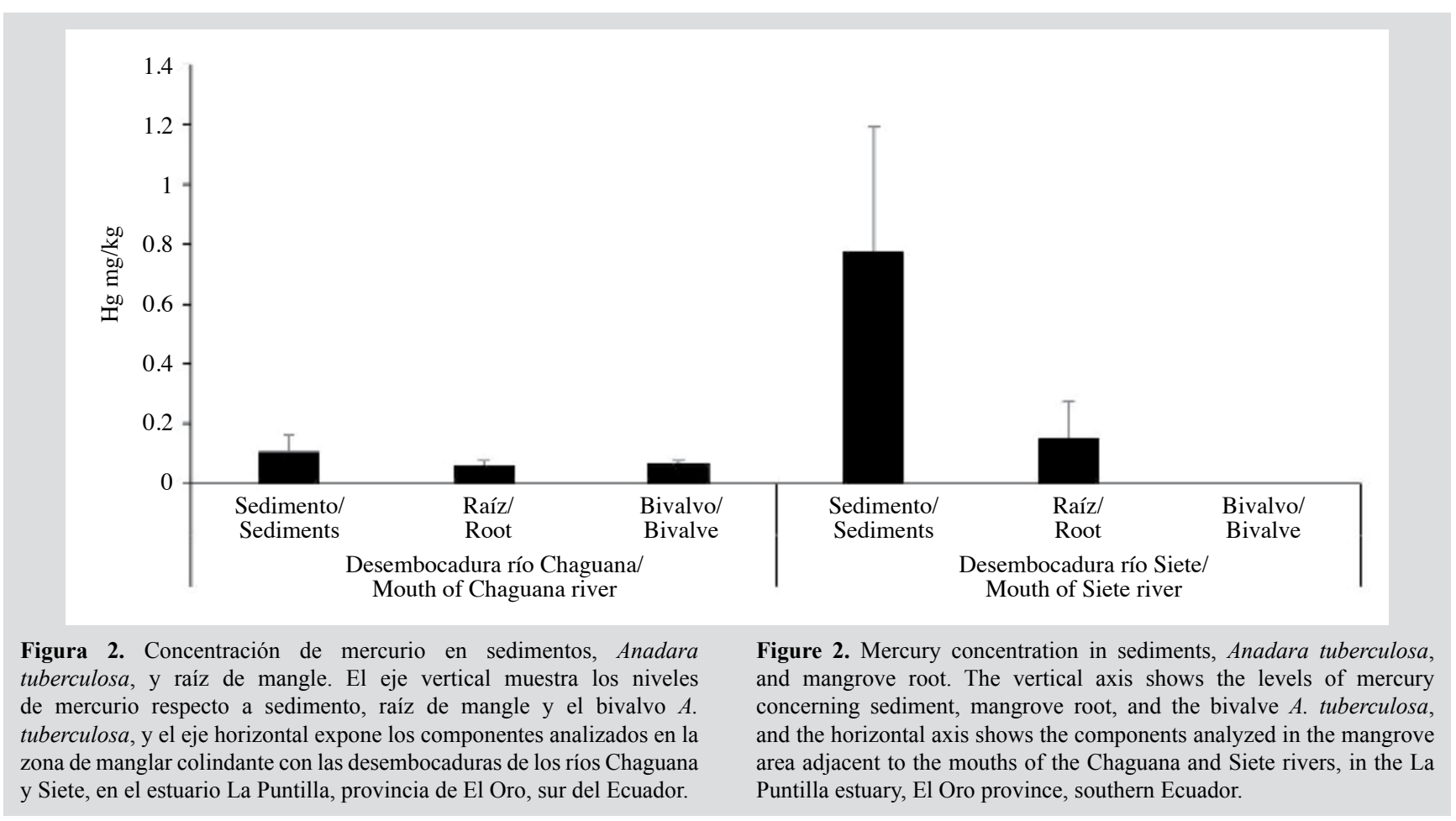


En el sector de manglar colindante con la desembocadura del río Chaguana se tomaron 29 muestras de sedimentos con niveles de concentración de mercurio de $0,12 \pm 0,06 \mathrm{mg} / \mathrm{kg}$. El valor determinado representa cinco muestras $(18,5 \%)$ del total de las muestras obtenidas, de tal forma que 24 muestras $(81,5 \%)$ registraron concentraciones bajo el límite de detección. Esto supone que la presencia de mercurio es inconstante en el área de manglar próxima a la desembocadura del río Chaguana. Por otra parte, al dirigirse hacia el sector de manglar que colinda con el río Siete, el ingreso al sitio fue difícil en bajamar por la excesiva sedimentación que existe en el lecho del estuario, formando una barrera que impide el acceso hacia el río. En el fango del lecho de manglar contiguo a la desembocadura del río Siete se tomaron cinco muestras de sedimento, con lo cual se identificó en todas las muestras presencia de mercurio cuyas concentraciones fluctuaron alrededor de $0,77 \pm 0,42 \mathrm{mg} / \mathrm{kg}$. El análisis de varianza muestra que la concentración de mercurio en sedimentos de la zona de manglar colindante al río Siete fue significativamente diferente a la concentración registrada para la zona de manglar colindante al río Chaguana $(\mathrm{p}>0,05)$. Adicionalmente, a diferencia de lo observado en la desembocadura del río Chaguana, en los sedimentos del área de manglar contigua al río Siete se determinó una distribución homogénea al registrarse la concentración más alta con $1,09 \mathrm{mg} / \mathrm{kg}$ de mercurio.

En lo que respecta a la concentración de mercurio en raíz de mangle en el río Chaguana, de un total de 23 muestras, cinco muestras $(21 \%)$ registraron una concentración de mercurio de $0,06 \pm 0,01 \mathrm{mg} / \mathrm{kg}$. En comparación, 18 muestras $(79 \%)$ registraron concentraciones bajo límite de detección. En contraste, en el río Siete, cinco muestras de raíz de mangle obtenidas reportaron una concentración de $0,151 \pm 0,121 \mathrm{mg} / \mathrm{kg}$. El análisis de varianza muestra que la concentración de mercurio en raíz de mangle en el área colindante a la desembocadura del río Chaguana no fue significativamente diferente a la concentración registrada cerca al río Siete $(\mathrm{p}>0,05)$.

En relación con A. tuberculosa, $60 \%$ de los individuos capturados tuvo un tamaño entre $3-4 \mathrm{~cm}, 31 \%$ entre $4-5 \mathrm{~cm}$ y $9 \%$ entre $5-5,5 \mathrm{~cm}$. Análogamente, los pesos con valvas de los individuos registrados fueron de $15,5 \mathrm{~g}$, 44,2 g y 78,7 g respectivamente según su tamaño. El peso del tejido muscular representó aproximadamente un $30 \%$ de su peso total, es decir, que varió entre 4,46-23,60 gramos de peso húmedo por individuo. La composición de una muestra se consiguió juntando entre 20 y 30 ejemplares
In the mangrove sector adjacent to the Chaguana river's mouth, 29 sediment samples were taken with mercury concentration levels of $0.12-0.06 \mathrm{mg} / \mathrm{kg}$. The determined value represents five samples $(18.5 \%)$ of the total samples obtained, in such a way that 24 samples $(81.5 \%)$ reported concentrations below the detection limit. This means that the presence of mercury is inconsistent in the mangrove area near the mouth of the Chaguana river. On the other hand, when heading towards the mangrove sector that adjoins the Siete river, entry to the site was difficult at sea due to the excessive sedimentation that exists in the estuary bed, forming a barrier that blocks access to the river. In the mud of the mangrove bed next to the Siete river's mouth, five sediment samples were taken, identifying in all the samples the presence of mercury whose concentrations fluctuated around $0.77-0.42 \mathrm{mg} / \mathrm{kg}$. The analysis of variance shows that the mercury concentration in sediments of the mangrove zone adjacent to the Siete river was significantly different from the level registered for the mangrove zone adjacent to the Chaguana river $(\mathrm{p}>0.05)$. Additionally, unlike what was determined for the mouth of the Chaguana river, a homogeneous distribution was established in the sediments of the mangrove area adjacent to the Siete river, registering the highest concentration with $1.09 \mathrm{mg} / \mathrm{kg}$ of mercury.

Regarding the concentration of mercury in mangrove root in the Chaguana river, out of a total of 23 samples, five samples $(21 \%)$ reported a mercury concentration of $0.06-0.01 \mathrm{mg} / \mathrm{kg}$. In comparison, 18 (79 \%) samples reported levels below the detection limit. In contrast, in the Siete river of five mangrove root samples obtained, reported a concentration of $0.151-0.121 \mathrm{mg} / \mathrm{kg}$. The analysis of variance shows that the level of mercury in mangrove root in the area adjacent to the mouth of the Chaguana river was not significantly different from the concentration registered near the Siete river $(p>0.05)$.

Concerning A. tuberculosa, $60 \%$ of the captured individuals had a size between $3-4 \mathrm{~cm}, 31 \%$ between 4-5 cm, and $9 \%$ fluctuated between $5-5.5 \mathrm{~cm}$. Similarly, the registered individuals' valve weights were $15.5 \mathrm{~g}$, $44.2 \mathrm{~g}$, and $78.7 \mathrm{~g}$, respectively, according to their size. The mass of the muscle tissue represented approximately $30 \%$ of its total weight; that is, it varied between 4.46 to $23.60 \mathrm{~g}$ of wet weight per individual. The composition of a sample was achieved by gathering between 20 to 30 specimens according to the individual's size. In the mangrove bed 
según el tamaño de los individuos. En el lecho de manglar hacia la desembocadura del río Chaguana se recolectaron 450 individuos con lo cual se completó un total de 15 muestras. De ellas, 14 registraron concentraciones por debajo del límite de detección y solo una reportó presencia de mercurio con una concentración de $0,034 \mathrm{mg} / \mathrm{kg}$. En contraste, en el lecho de manglar hacia la desembocadura del río Siete la escasa presencia del bivalvo A. tuberculosa imposibilitó completar una muestra del peso requerido para el análisis, coincidiendo con la presencia de algunos ejemplares muertos en el hábitat.

Análisis de riesgo ecológico y riesgo sanitario ambiental

La Tabla 3 muestra los valores de los índices de riesgo ecológico para los escenarios A y B. En el sector hacia el río Siete, se estimó que el Cf está en la categoría "considerable", el Er es considerado "alto" mientras que el RI está en un nivel "moderado". En contraste, hacía la zona del estuario que conecta la desembocadura del río Chaguana, los factores Cf, Er y el índice RI se situaron en la categoría "bajo". towards the mouth of the Chaguana river, 450 individuals were collected, completing a total of 15 samples. Of these, 14 samples reported concentrations below the detection limit and only one with a level of $0.034 \mathrm{mg} / \mathrm{kg}$ of mercury. In contrast, in the mangrove bed towards the mouth of the Siete river, the scarce presence of $A$. tuberculosa made it impossible to complete a sample at the required weight for the analysis, coinciding with the presence of some dead specimens in the habitat.

\section{Ecological risk and environmental health risk analysis}

Table 3 shows the values of the ecological risk indices for scenarios A and B. The results demonstrated that in the Siete river the $\mathrm{Cf}$ is in the "considerable" category, the Er is "high" while the RI it is at a "moderate" level. In contrast, the Factors $\mathrm{Cf}$, Er, and the RI index were located in the "low" category at the mouth of the Chaguana river.

Tabla 3. Cálculo de los índices de riesgo ecológico.

Table 3. Calculation of ecological risk indices.

\begin{tabular}{|l|c|c|c|}
\hline \multicolumn{1}{|c|}{ Variables [adimensionales/dimensionless] } & Abreviatura/ & Escenario A/Stage A & Escenario B/Stage B \\
\cline { 3 - 4 } & Abbreviation & $\begin{array}{c}\text { Línea de costa sector } \\
\text { río Siete/Coastline sector } \\
\text { Seven river }\end{array}$ & $\begin{array}{c}\text { Línea de costa sector } \\
\text { río Chaguana/Coastline sector } \\
\text { Chaguana river }\end{array}$ \\
\hline $\begin{array}{l}\text { Factor de contaminación/Pollution factor } \\
\text { Factor de potencial riesgo ecológico/Potential ecological risk factor }\end{array}$ & $\mathrm{Cf}$ & 4.34 & 0.9 \\
Índice de riesgo ecológico/Ecological risk index & $\mathrm{Er}$ & 173.76 & 36 \\
\hline
\end{tabular}

El presente estudio define un modelo que considera los sedimentos como vía de exposición por contacto dérmico, y el tejido blando de $A$. tuberculosa como vía de exposición por ingestión. El contacto dérmico está asociado a la manipulación de sedimento y del bivalvo contaminado por mercurio. Esto sucede por absorción percutánea del elemento que puede ingresar al cuerpo humano al estar en contacto con elementos posiblemente contaminados. Los receptores considerados corresponden a adultos y niños que habitan en el sector o realizan faneas de pesca en los bosques del manglar (Figura 3 ).
This study defines a model that considers sediments as the route of exposure by dermal contact, and the soft tissue of $A$. tuberculosa as the route of exposure by ingestion. Dermal contact is associated with the manipulation of sediment and mercury-contaminated bivalve. That is percutaneous absorption of the element that can enter the human body when a person is in touch with the contaminant. The receivers considered correspond to adults and children who live in the sector or make fishing lines in the mangrove forest (Figure 3). 


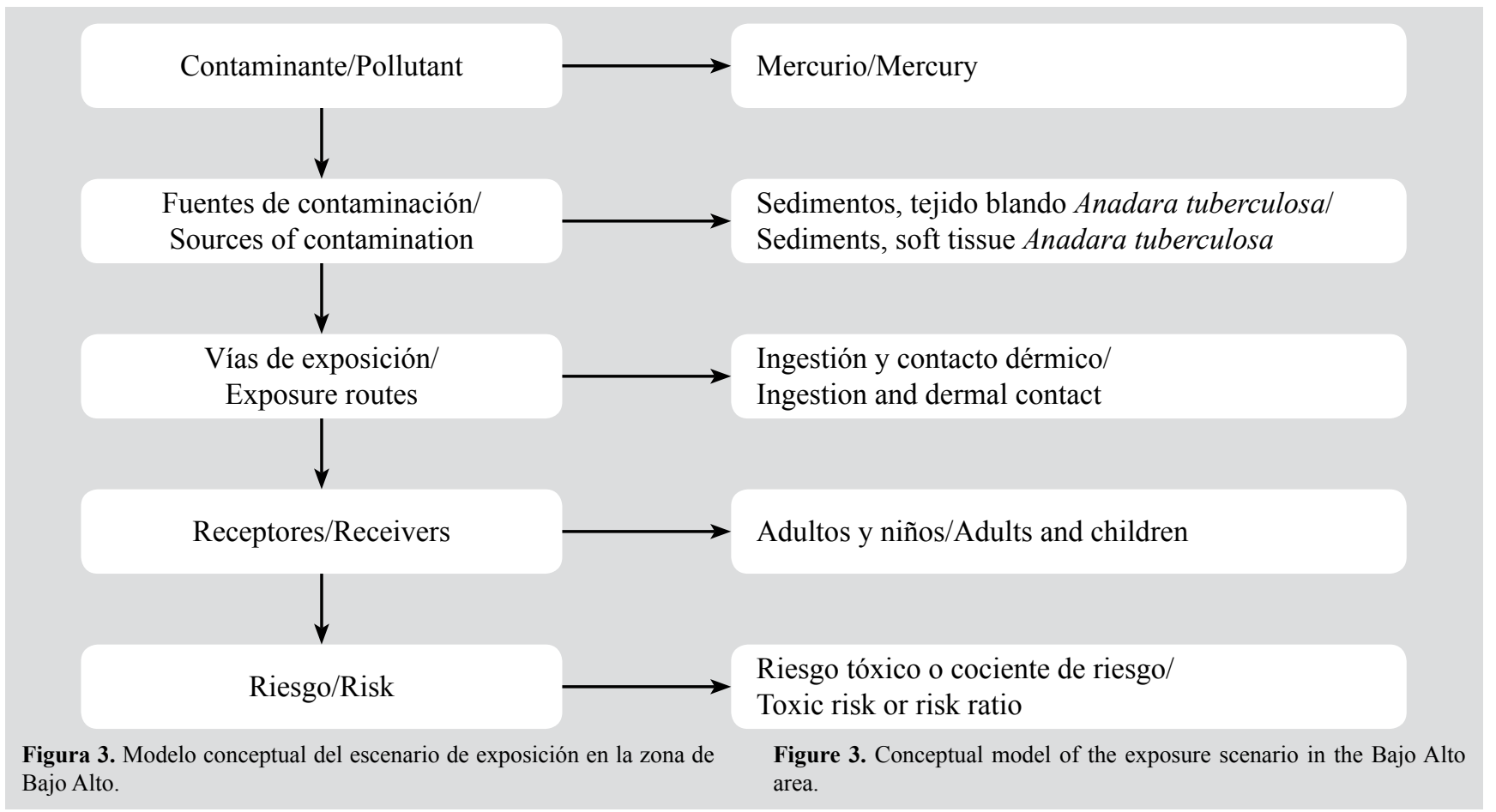

El análisis de riesgo sanitario ambiental fue realizado designando valores a los parámetros mostrados en las ecuaciones 4-7 considerando los factores y valores mostrados en la Tabla 4. Los datos de concentración de mercurio en sedimentos para el río Chaguana y el río Siete correspondieron a $0,225 \mathrm{mg} / \mathrm{kg}$ y $1,086 \mathrm{mg} / \mathrm{kg}$, respectivamente, estimados a través del criterio UCL de la EPA (U.S. Environmental Protection Agency, 2002). Los valores de cálculo del riesgo en el escenario A, correspondiente al hábitat de manglar colindante a la desembocadura del río Siete para adultos y niños, fueron $2,00 \times 10^{-02}$ y $1,74 \times 10^{-01}$, respectivamente; en cambio, para el escenario $\mathrm{B}$, correspondiente al hábitat de manglar colindante al río Chaguana, los valores para adultos y niños son $1,83 \times 10^{-02}$ y $1,02 \times 10^{-01}$, respectivamente.
The environmental health risk analysis was carried out designating values to the parameters shown in Equations 4-7, considering the factors and levels shown in Table 4. The data of mercury concentration in sediments for the Chaguana river and the Siete river corresponds to $0.225 \mathrm{mg} / \mathrm{kg}$ and $1.086 \mathrm{mg} / \mathrm{kg}$, respectively, estimated using the UCL criteria of the EPA (US Environmental Protection Agency, 2002). The risk calculation values for scenario A corresponds to the mangrove habitat adjacent to the mouth of the Siete river for adults, and children were $2.00 \times 10^{-02}$ and $1.74 \times 10^{-01}$, respectively. On the other hand, for scenario $\mathrm{B}$ corresponding to the mangrove habitat adjacent to the Chaguana river, the values for adults and children are $1.83 \times 10^{-02}$ and $1.02 \times 10^{-01}$, respectively.
Tabla 4. Valores obtenidos del cálculo del Hazard Quotient para adultos y niños para los escenarios 1 y 2 .
Table 4. Values obtained from the calculation of the Hazard Quotient for adults and children for scenarios 1 and 2 .

\begin{tabular}{|l|c|c|c|c|}
\hline \multirow{2}{*}{$\begin{array}{l}\text { Vías de exposición/ } \\
\text { Routes of exposure }\end{array}$} & \multicolumn{2}{|c|}{$\begin{array}{c}\text { Línea de costa sector río Siete/ } \\
\text { Coastline sector Seven river }\end{array}$} & \multicolumn{2}{|c|}{$\begin{array}{c}\text { Línea de costa sector río Chaguana/ } \\
\text { Coastline sector Chaguana river }\end{array}$} \\
\cline { 2 - 5 } & $\begin{array}{c}\text { HQ adultos (adimensional)/ } \\
\text { HQ adults (dimensionless) }\end{array}$ & $\begin{array}{c}\text { HQ niños (adimensional)/ } \\
\text { HQ children (dimensionless) }\end{array}$ & $\begin{array}{c}\text { HQ adultos (adimensional)/ } \\
\text { HQ adults (dimensionless) }\end{array}$ & $\begin{array}{c}\text { HQ niños (adimensional)/ } \\
\text { HQ children (dimensionless) }\end{array}$ \\
\hline $\begin{array}{l}\text { Ingestión de sedimentos/ } \\
\text { Sediment ingestion }\end{array}$ & $1.74 \times 10^{-02}$ & $1.62 \times 10^{-01}$ & $3.60 \times 10^{-03}$ & $3.36 \times 10^{-02}$ \\
$\begin{array}{l}\text { Contacto dérmico por sedimentos/ } \\
\text { Dermal contact by sediment }\end{array}$ & $2.63 \times 10^{-03}$ & $1.23 \times 10^{-02}$ & $5.45 \times 10^{-04}$ & $2.54 \times 10^{-03}$ \\
\hline $\begin{array}{l}\text { Ingestión tejido blando } \\
\text { Anadara tuberculosa }\end{array}$ & - & - & $1.42 \times 10^{-02}$ & $6.61 \times 10^{-02}$ \\
Hazard Quotient Total & $2.00 \times 10^{-02}$ & $1.74 \times 10^{-01}$ & $1.83 \times 10^{-02}$ & $1.02 \times 10^{-01}$ \\
\hline
\end{tabular}


A partir de los resultados obtenidos sobre la estimación del riesgo total para adultos y niños, se observa que los límites de tolerancia del HQ = 1 no son superados en ninguno de los dos sitios. Sin embargo, los valores registrados en el río Chaguana son significativamente menores a los determinados en los sitios cercanos al río Siete.

\section{DISCUSIÓN}

El presente trabajo analizó las concentraciones de mercurio en tres componentes del hábitat de manglar en el estuario de La Puntilla, al sur del Ecuador. Se estimó el riesgo ecológico y sanitario-ambiental del mercurio en dos sitios. Es importante resaltar que las muestras fueron tomadas en el hábitat del manglar y no precisamente en el lecho del estuario. Por tanto, las muestras de sedimentos representan al fango que caracteriza el bioma de manglar colindante a las desembocaduras de los ríos Chaguana y Siete.

De acuerdo con los resultados obtenidos se observa que los mecanismos de transporte y difusión de mercurio hacia el estuario La Puntilla se realizan por drenaje fluvial desde la parte alta donde se descargan desechos y relaves de minería que alcanzan la zona costera. Estudios previos sobre la concentración de mercurio en sedimentos del lecho del río Siete reportaron concentraciones de mercurio de $2,0 \mathrm{mg} / \mathrm{kg}$ (Tarras-Wahlberg et al., 2000), $13 \mathrm{mg} / \mathrm{kg}$ (Appleton et al., 2001) y $1,4 \mathrm{mg} / \mathrm{kg}$ (Carling et al., 2013). En el estuario del río Tumbes, Schudel et al. (2018) determinaron niveles de $0,13 \mathrm{mg} / \mathrm{kg}$ de mercurio en sedimentos y mediante análisis isotópico confirmaron que el elemento está asociado a descargas de material de desecho de actividades mineras agua arriba del río Puyango-Tumbes. Alta variabilidad en las concentraciones de mercurio se observó en los tres componentes del río Chaguana con el $80 \%$ de muestras bajo limite detección. Esto puede deberse a múltiples factores tales como la mezcla y dilución de agua en el estuario, así como también a la dinámica intermareal que caracteriza la zona costera (Tam y Wong, 1995). Aproximadamente a $20 \mathrm{~km}$ del área de estudio, se han reportado concentraciones de 3,97 $\pm 0,73 \mathrm{mg} / \mathrm{kg}$ de mercurio en sedimentos (Marin et al., 2016). Lacerda et al. (1993), al investigar manglares en una zona litoral de Brasil, evidenció mayor concentración de metales en sedimentos del lecho del estuario que en el manglar. Similarmente, Silva et al. (2003) determinó concentraciones de mercurio entre $0,022-0,060 \mathrm{mg} / \mathrm{kg}$ y corroboró que este elemento se acumula mayormente en los sedimentos del lecho del estuario en comparación con los depósitos del bosque de manglar. Cuando el mercurio entra
From the results obtained, estimating the total risk for adults and children, it is observed that the tolerance limits of $\mathrm{HQ}=1$, were not exceeded in the two sites. However, the values recorded in the Chaguana river are significantly lower than those determined in the Siete river.

\section{DISCUSSION}

The present work analyzed mercury concentrations in three components of the mangrove habitat in the La Puntilla estuary in southern Ecuador. We estimated the ecological and environmental health risk of mercury at two sites. It is essential to highlight that the samples were taken in the mangrove habitat and not precisely in the riverbed. Hence, the sediment samples represent the mud that characterizes the mangrove biome adjacent to the Chaguana river's mouths.

According to the results obtained, it is observed that the transport and diffusion mechanisms of mercury towards the La Puntilla estuary are carried out by river drainage from the upper part where waste and mining tailings are discharged reaching the coastal zone. Previous studies on the concentration of mercury in sediments of the riverbed Siete reported mercury concentrations of $2.0 \mathrm{mg} / \mathrm{kg}$ (Tarras-Wahlberg et al., 2000), $13 \mathrm{mg} / \mathrm{kg}$ (Appleton et al., 2001) and $1.4 \mathrm{mg} / \mathrm{kg}$ (Carling et al., 2013). In the estuary of the Tumbes river, Schudel et al., (2018) determined levels of $0.13 \mathrm{mg} / \mathrm{kg}$ of mercury in sediments and through isotopic analysis confirm that the element is associated with discharges of waste material from mining activities upstream of the river PuyangoTumbes. High variability in mercury concentrations was observed in the three components of the Chaguana river, with $80 \%$ of samples under the detection limit. This may be due to multiple factors such as water mixing and dilution in the estuary zone, as well as the dynamics that characterize the coastal area (Tam and Wong, 1995). Approximately $20 \mathrm{~km}$ from our study area, concentrations of $3.97 \pm 0.73 \mathrm{mg} / \mathrm{kg}$ of mercury have been reported in sediments (Marin et al., 2016). Lacerda et al. (1993), when investigating mangroves in a coastal zone of Brazil, found higher concentration of metals in sediments from the estuary bed than in mangroves mudflats. Similarly, Silva et al. (2003) determined mercury concentrations between $0.022-0.060 \mathrm{mg} / \mathrm{kg}$ of mercury and corroborates that mercury accumulates mostly in sediments from the estuary bed compared to deposits from the mangrove forest. When mercury enters aquatic environments, it precipitates due to 
en ambientes acuáticos, se precipita debido a su alta densidad y se incorpora en el fondo, que actúa como sumidero donde se adhiere a las partículas más finas (Andren y Harriss, 1975; Vane et al., 2009). En virtud de lo examinado, se requieren estudios más puntuales para comprender los mecanismos que gobiernan la movilidad del mercurio en el bioma de manglar del río Siete y sus alrededores.

Las concentraciones de mercurio encontradas en el fango del manglar muestran superioridad con relación a los limites estándares de Canadá para la protección de la vida acuática que está en $0,49 \mathrm{mg} / \mathrm{kg}$. Adicionalmente, las concentraciones de mercurio detectadas en el presente estudio superan los niveles de remediación (Soil Cleanup Target Levels) establecidos por el Departamento de Ambiente y Toxicología de la Universidad de Florida (Center for Environmental \& Human Toxicology, 2005). Estos criterios están basados en la lixiviación de contaminantes sobre agua superficial marina (Leachability Based on Marine Surface Water Criteria) que establece un límite de $0,03 \mathrm{mg} / \mathrm{kg}$. La legislación ecuatoriana no establece criterios de remediación de sedimentos para ambientes de manglar. El hábitat de manglar caracteriza aproximadamente el $75 \%$ de la línea costera del Ecuador, y representa una importante ecorregión para el crecimiento y la reproducción de algunas especies marinas de peces, moluscos y crustáceos. En el presente estudio, la muestra de raíz de mangle contenía material particulado, microalgas e invertebrados, lo cual supone una posible contaminación de peces marinos que en marea alta o pleamar se acercan al manglar para alimentarse. En el río Chaguana, la concentración de mercurio en sedimentos fue aproximadamente dos veces superior a la concentración de mercurio en raíz de mangle. En el río Siete, la diferencia fue ocho veces superior. Esto sugiere mayor absorción de mercurio en sedimentos y una distribución menor de mercurio en el sistema radicular de los manglares en relación con los sedimentos (Silva et al., 2003; Alongi, 2005). Aunque, la investigación sobre mercurio en la raíz de mangle es limitada, Huang et al. (2020) discute procesos de absorción de mercurio en raíz de mangle e indica que los mecanismos de transporte pueden estar sujetos a las variaciones intermareales. El mercurio adherido a las raíces aéreas del manglar puede sufrir un proceso de volatilización por efecto de la radiación solar en el momento de exposición a la atmósfera en marea baja (Huang et al., 2020).

En relación con la contaminación por mercurio en bivalvos, se conoce que en un mismo lugar la concentración difiere entre diferentes especies e individuos (Elder y Collins, its high density and is incorporated into the bottom, which acts as a sink that adheres to the finest particles (Andren and Harriss, 1975; Vane et al., 2009). Based on what was examined in this study, more specific studies are required to understand the mechanisms that govern mercury's mobility in the mangrove biome of the Siete river and its surroundings.

The mercury concentrations found in the mangrove mud show superiority to the Canadian standard limits for the protection of aquatic life, which is $0.49 \mathrm{mg} / \mathrm{kg}$. Additionally, the mercury concentrations detected in the present study exceed the remediation levels (Soil Cleanup Target Levels) established by the Department of Environment and Toxicology of the University of Florida (Center for Environmental \& Human Toxicology, 2005). This criterion is based on Leachability Based on Marine Surface Water Criteria, which establishes a limit of $0.03 \mathrm{mg} / \mathrm{kg}$. Ecuadorian legislation does not establish sediment remediation criteria for mangrove environments. The mangrove forest habitat characterizes approximately $75 \%$ of Ecuador's coastline, representing a critical ecoregion for the growth and reproduction of some marine species of fish, mollusks, and crustaceans. In the present study, the mangrove root sample contained particulate material, microalgae, and invertebrates, which supposes possible contamination of marine fish that at low tide or high tide graze the mangrove to feed. In the Chaguana river, the concentration of mercury in sediments was approximately two times higher than the mercury level in mangrove roots. In the Siete river, the difference was eight times higher. This suggests higher absorption of mercury in sediments, while lower mercury distribution in the mangrove root system (Silva et al., 2003; Alongi, 2005). Although research on mercury in mangrove root is limited, Huang et al. (2020) discusses mercury absorption processes in mangrove root and indicates that the transport mechanisms may be subject to intertidal variations. The mercury attached to the mangrove aerial roots may undergo a volatilization process due to the effect of solar radiation on the moment of exposure to the atmosphere at low tide (Huang et al., 2020).

Concerning mercury contamination in bivalves, it is known that the concentration in the same place differs between different species and individuals (Elder and Collins, 1991), and the age of the organisms can influence the accumulation of mercury (Otchere et al., 2003). Studies in mangroves in India determined a low 
1991), pudiendo la edad de los organismos influir en la acumulación de mercurio (Otchere et al., 2003). Estudios en manglares de la India determinaron baja correlación entre la concentración de mercurio en sedimentos y el determinado en el gasterópodo Pirinella cingulata. La investigación sobre el mercurio en bivalvos en los últimos 50 años ha sido revisada por Otchere (2019), quien reporta una extensa lista de moluscos bivalvos de varias localidades y diversos ambientes cuyas concentraciones promedio fluctúan alrededor de $0,92 \pm 1,67 \mathrm{mg} / \mathrm{kg}$, con un valor mínimo de $0,03 \mathrm{mg} / \mathrm{kg}$ y máximo de $7,5 \mathrm{mg} / \mathrm{kg}$. Los valores registrados en el presente estudio en las muestras de la concha negra $A$. tuberculosa obtenidas en el manglar contiguo al río Chaguana están cerca de valores mínimos reportados en otros estudios. Estos niveles no se consideran altos en referencia a los límites permisibles de la Organización de las Naciones Unidas para la Agricultura y la Alimentación (FAO) y la Organización Mundial de la Salud (OMS), que presentan un valor de referencia de $0,5 \mathrm{mg} / \mathrm{kg}$ (World Health Organization, 2008). Es importante considerar que una muestra estuvo compuesta por 20 a 30 individuos, lo que supone variabilidad en la concentración de mercurio entre los especímenes que forman una muestra correspondiente a una misma especie en una misma localidad. Consecuentemente, un solo ejemplar podría reportar concentraciones entre 0,68 a $1,02 \mathrm{mg} / \mathrm{kg}$, conforme al rango manifestado por Otchere (2019).

Se ha reportado que el mercurio puede ocasionar alteración en células sanguíneas de moluscos bivalvos pudiendo a ciertas concentraciones tener efecto letal en organismos acuáticos (Jakimska et al., 2011; Denil et al., 2017). Otros investigadores deducen la presencia de mecanismos de defensa frente a los metales a través de vesículas que facilitan la excreción celular (Dallinger, 1993; Claisse et al., 2001), o mediante una protección a través de sus valvas como biopelículas de protección (Scardino et al., 2013). En ese mismo orden, otros estudios afirman que la concha externa tiene mayor capacidad de absorber el metal del agua o sedimento alcanzando concentraciones incluso más altas que las de los tejidos internos (Zuykov et al., 2011, 2012). Adicionalmente, factores biológicos tales como especie, edad, tamaño, sexo, genotipo, fenotipo, actividad de alimentación y estado reproductivo influyen en la acumulación de metales y su toxicidad (Riget et al., 1996; Kehrig et al., 2006; Sevillano-Morales et al., 2015), aspectos que requieren más atención en la zona de estudio.

El estuario La Puntilla es una zona dinámica influenciada por mareas que provienen del océano y por correlation between the concentration of mercury in sediments and that established in the gastropod Pirinella cingulata. Research on mercury in bivalves in the past 50 years has been reviewed by Otchere (2019), who reports an extensive list of bivalve molluscs from various locations and diverse environments whose average concentrations fluctuate around $0.92 \pm 1.67 \mathrm{mg} / \mathrm{kg}$, with a minimum value of $0.03 \mathrm{mg} / \mathrm{kg}$ and a maximum of $7.5 \mathrm{mg} / \mathrm{kg}$. The values recorded in the present study in the samples of the black shellfish $A$. tuberculosa obtained in the mangrove mudflats next to the Chaguana river are close to the minimum values reported in other studies. These levels are not considered high about the permissible limits of the Food and Agriculture Organization (FAO) and the World Health Organization (WHO), which presents a reference value of $0.5 \mathrm{mg} / \mathrm{kg}$ (World Health Organization, 2008). It is essential to consider that a sample consisted of 20 to 30 individuals, which implies variability in the concentration of mercury between the specimens that form one sample size corresponding to the same species in the same locality. Consequently, a single specimen could report mercury levels between 0.68 to $1.02 \mathrm{mg} / \mathrm{kg}$, which are similar to the range stated by Otchere (2019).

It has been reported that mercury can cause a cellular alteration in blood cells of bivalve molluses, and an absolute concentration can have a lethal effect in aquatic organisms (Jakimska et al., 2011; Denil et al., 2017). Other researchers deduce the presence of protection mechanisms against metals through vesicles that facilitate cell excretion (Dallinger, 1993; Claisse et al., 2001), or defense throughout their shells as protective biofilms (Scardino et al., 2013). In the same order, other studies affirm that the outer shell can absorb metal from water or sediment, reaching concentrations higher than those of the internal tissues (Zuykov et al., 2011, 2012). Additionally, biological factors such as species, age, size, sex, genotype, phenotype, feeding activity, and reproductive status influence the accumulation of metals and their toxicity (Riget et al., 1996; Kehrig et al., 2006; Sevillano-Morales et al., 2015), aspects that require more attention in the study area.

La Puntilla estuary is a dynamic area influenced by tides that come from the ocean and by the drainage of freshwater from rivers. In southern Ecuador the estuary dynamics is associated to an intensive coastal aquaculture. In the Siete river, a fluvial body of low flow was observed; therefore, a more severe effect is presumed in the 
el drenaje de agua dulce de los ríos. En el sur del Ecuador, la dinámica del estuario está asociada con una intensa acuacultura costera. En el río Siete se observó un cuerpo fluvial de escaso caudal; por tanto, se presume un efecto más severo en la configuración del cauce (Figura 3a). En contraste, en el río Chaguana (Figura 3b) se observó un cuerpo de agua más saludable, donde se observa la vida acuática y silvestre de algunas especies incluyendo aves, bivalvos y crustáceos. La percepción de los pescadores artesanales ante la situación observada en el estuario es negativa y crítica. Ellos demuestran preocupación por el escaso caudal del río y los desechos de la actividad minera aguas arriba. Así mismo, de manera general, asocian los problemas del hábitat a las descargas de aguas de desecho de las granjas camaroneras. Con especial cuidado al río Siete, los pescadores no se acercan al lugar indicando que $A$. tuberculosa y otras especies bioacuáticas tales como el cangrejo rojo son imperceptibles en esa zona.

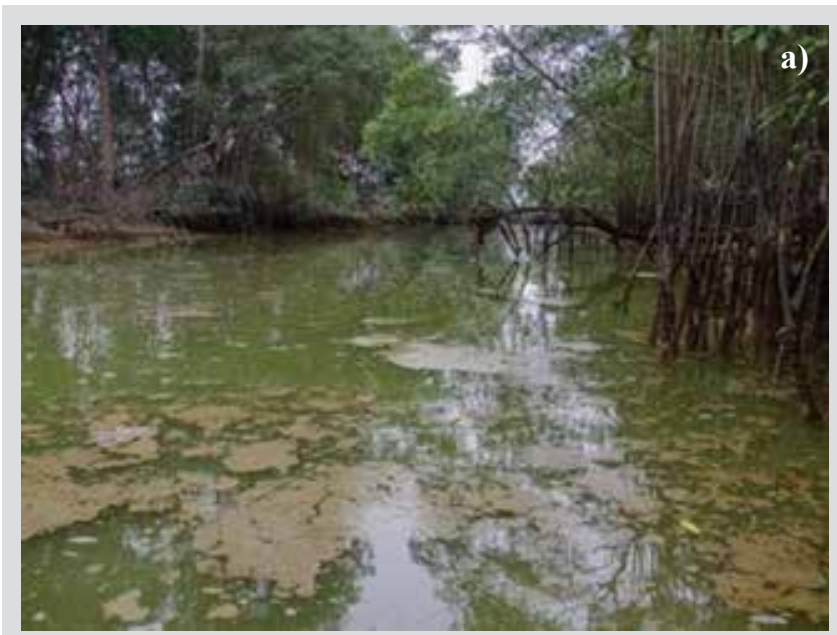

Figura 3. Imágenes de la zona de estudio donde se realizaron los muestreos a) río Siete b) río Chaguana. configuration of the channel (Figure 3a). In contrast, in the Chaguana river (Figure 3b), a healthier body of water was observed, where the aquatic and wildlife of some species are found, including the presence of birds, bivalves and crustaceans. The perception of artisanal fishers regarding the situation observed in the estuary is negative and critical. It indicates their concern about the river's low flow and the waste from the upstream mining activity. Likewise, they generally associate habitat problems with wastewater discharges from shrimp farms. With special precaution to the Siete river, the fishermen do not approach the place, indicating that $A$. tuberculosa and other bio-aquatic species such as the red crab are invisible in that area.

From the results obtained, mercury contamination in the mangrove swamp of the La Puntilla estuary defines two risk scenarios, referred to as scenario $A$ at the mouth of

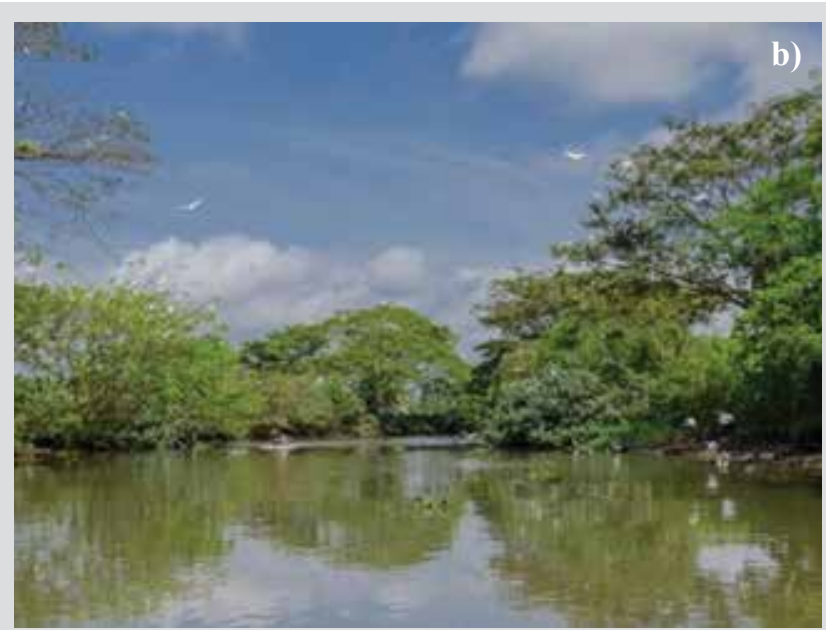

Figure 3. Images of the study area where the samples were taken a) Siete river b) Chaguana river.
De los resultados obtenidos, la contaminación por mercurio en el manglar del estuario La Puntilla define dos escenarios de riesgo, referidos como escenario A (desembocadura del río Siete) y escenario B (desembocadura del río Chaguana). Para ninguno de los casos se superó el HQ, que presentó valores inferiores a 1. Sin embargo, es importante considerar que el HQ no es más que una estimación conservadora de los efectos potenciales para la salud humana. Los factores de riesgo e índices de riesgo ecológico, asociados al río Siete, son aproximadamente cuatro veces mayores que en el río Chaguana. La escasa presencia de A. tuberculosa en la desembocadura del río Siete demuestra un efecto ecológico severo en este cuerpo the Siete river and Scenario B at the mouth of the Chaguana river. For none of the cases was the HQ exceeded, presenting values less than one. However, it is crucial to consider that the HQ is nothing more than a conservative estimate of the potential effects on human health. The risk factors and ecological risk indices associated with the Siete river are approximately four times higher than in the Chaguana river. The scarce presence of A.tuberculosa at the mouth of the Siete river evidences a severe ecological effect on this stream. It causes local uncertainty, which is why it is necessary to deepen the research at this site. Other metals reported in the Siete river watershed that could add toxic effects to aquatic organisms were not 
fluvial y es motivo de incertidumbre local, por lo cual se requiere profundizar en la investigación en este sitio. La presencia de otros metales que se han reportado en la cuenca hídrica del río Siete y que podrían sumar efectos tóxicos para los organismos acuáticos no fue motivo de análisis en el presente trabajo. Es substancial ejecutar un programa de investigación integral en áreas de manglar considerando el análisis de otros metales y parámetros abióticos en el ecosistema.

\section{AGRADECIMIENTOS}

El presente trabajo se desarrolló a través del acuerdo de cooperación entre la Universidad Técnica de Machala-Ecuador y el Canadian International Resource and Develoment Institute (CIRDI) para la ejecución de los proyectos "TransMAPE" y "Contaminación por metales pesados". Se deja especial reconocimiento a los pescadores artesanales de la parroquia Tendales, en especial a la familia Guerrero-García, por su apoyo y acompañamiento en las faenas de campo y por el interés mostrado en la situación ecológica del lugar en el que habitan y trabajan. a reason for analysis in this study. It is substantial to execute a comprehensive research program in mangrove areas considering the analysis of other metals and abiotic parameters in the ecosystem.

\section{ACKNOWLEDGEMENTS}

This work was developed through the cooperation agreement between the Technical University of MachalaEcuador and the Canadian International Resource and Development Institute (CIRDI) for the execution of the "TransMAPE" and "Heavy metal pollution" projects. Special recognition is left to the artisanal fishermen of the Tendales parish, especially to the Guerrero-García family for their support and accompaniment in-field tasks, and interest demonstrated in the ecological situation of their place of living and work.

\section{BIBLIOGRAFÍA/LITERATURE CITED}

Alongi, D.M. 2013. Mangrove-microbe-soil relations. Interactions between macro- and microorganisms in marine sediments: 85-103. https://doi.org/10.1029/ CE060p0085

Andren, A.W. and R.C. Harriss. 1975. Observations on the association between mercury and organic matter dissolved in natural waters. Geochim. Cosmochim. Acta, 39: 1253-1258. https://doi.org/10.1016/0016-7037(75)90132-5.

Appleton, J.D., T.M. Williams, H. Orbea and M. Carrasco. 2001. Fluvial contamination associated with artisanal gold mining in the Ponce Enriquez, Portovelo-Zaruma and Nambija areas, Ecuador. Water Air Soil Poll., 131(1-4): 19-39.

Bazzi, A. 2014. Heavy metals in seawater, sediments and marine organisms in the Gulf of Chabahar, Oman Sea. J. Oceanogr. Mar. Sci., 5(3): 20-29. https:// doi.org/10.5897/JOMS2014.0110.

Cardoso, P.G., A.I. Lillebø, E. Pereira, A.C. Duarte and M.A. Pardal. 2009. Different mercury bioaccumulation kinetics by two macrobenthic species: the bivalve Scrobicularia plana and the polychaete Hediste diversicolor. Mar. Environ. Res., 68(1): 12-18. https://doi.org/10.1016/j.marenvres.2009.03.006.

Carling, G.T., X. Diaz, M. Ponce, L. Perez, L. Nasimba, E. Pazmino and W.P. Johnson. 2013. Particulate and dissolved trace element concentrations in three southern Ecuador rivers impacted by artisanal gold mining. Water Air Soil Poll., 224(2): 1415.

Center for Environmental \& Human Toxicology. 2005. Final technical report: Development of Cleanup Target Levels (CTLs). Division of Waste Management FDEP.

Chen, C.Y., M. Dionne, B.M. Mayes, D.M. Ward, S. Sturup and B.P. Jackson. 2009. Mercury bioavailability and bioaccumulation in estuarine food webs in the Gulf of Maine. Environ. Sci. Technol., 43(6): 1804-10. https://doi.org/10.1021/es8017122.

Claisse, D., D. Cossa, J. Bretaudeau-Sanjuan, G. Touchard and B. Bombled. 2001. Methylmercury in molluscs along the French coast. Mar. Pollut. Bull., 42(4): 329-332. https://doi.org/10.1016/S0025-326X(01)00036-4.

Dallinger, R. 1993. Strategies of metal detoxification in terrestrial invertebrates: 245-289. In Ecotoxicology of metals in invertebrates. Lewis Publishers, London.

Denil, D.J., F.F. Ching and J. Ransangan. 2017. Health risk assessment due to heavy metals exposure via consumption of bivalves harvested from Marudu Bay, Malaysia. Open J. Mar. Sci., 7: 494-510. https://doi.org/10.4236/ojms.2017.74035. 
Elder, J.F. and J.J. Collins. 1991. Freshwater molluscs as indicators of bioavailability and toxicity of metals in surface-water systems. Rev. Environ. Contam. Toxicol., 122: 37-79. https://doi.org/10.1007/978-1-4612-3198-1_2.

Environmental Protection Agency. 1997. Analysis of risk for environmental pollutants application of deterministic and probabilistic methods for a school scenario.

Hakanson, L. 1980. An ecological risk index for aquatic pollution control. A sedimentological approach. Water Res., 14(8): 975-1001. https://oi. org/10.1016/0043-1354(80)90143-8.

Huang, S., R. Jiang, Q. Song, Y. Zhang, Q. Huang, B. Su, ... and H. Lin. 2020. Study of mercury transport and transformation in mangrove forests using stable mercury isotopes. Sci. Total Environ., 704, 135928.

Institute of Marine and Coastal Research. 2013. Manual of analytical techniques for the determination of isicochemical parameters and marine pollutants (water, sediments and organisms). Inst. Coast. Mar. Res., Santa Marta. https://doi.org/10.1017/ CBO9781107415324.004.

Jakimska, A., P. Konieczka, K. Skóra and J. Namieśnik. 2011. Bioaccumulation of metals in tissues of marine animals, Part I: the role and impact of heavy metals on organisms. Pol. J. Environ. Stud., 20(5): 1117-1125.

Kehrig, H. A., M. Costa, I. Moreira and O. Malm. 2006. Total and methyl mercury in different species of molluscs from two estuaries in Río de Janeiro state. J. Braz. Chem. Soc., 17(7): 1409-1418.

Lacerda, L.D., C.E. Carvalho, K. Tanizaki, A. Ovalle and C. Rezende. 1993. The biogeochemistry and trace metals distribution of mangrove rhizospheres. Biotropica, 252-257.

Le, D.Q., K. Tanaka, L.V. Dung, Y.F. Siau, L. Lachs, S.T. Kadir, ... and K. Shirai. 2017. Biomagnification of total mercury in the mangrove lagoon foodweb in east coast of Peninsula, Malaysia. Reg. Stud. Mar. Sci., 16, 49-55. https://doi.org/10.1016/j.rsma.2017.08.006

Maanan, M. 2008. Heavy metal concentrations in marine molluscs from the Moroccan coastal region. Environ. Poll., 153(1): 176-183. https:// doi. org/10.1016/j.envpol.2007.07.024.

Marchand, C., E. Lallier-Vergès, F. Baltzer, P. Albéric, D. Cossa and P. Baillif. 2006. Heavy metals distribution in mangrove sediments along the mobile coastline of French Guiana. Mar. Chem., 98(1): 1-17. https://doi.org/10.1016/j.marchem.2005.06.001.

Marín, A., V.H. González, B. Lapo, E. Molina y M. Lemus. 2016. Niveles de mercurio en sedimentos de la zona costera de El Oro, Ecuador. Gayana, 80(2): 147-153. http://dx.doi.org/10.4067/S0717-65382016000200147

Medina, M.H., J. Correa and C. Barata. 2007. Micro-evolution due to pollution: possible consequences for ecosystem responses to toxic stress. Chemosphere, 67(11): 2105-2114. https://doi.org/10.1016/j.chemosphere.2006.12.024.

Morel, F.M., A. Kraepiel and M. Amyot. 1998. The chemical cycle and bioaccumulation of mercury. Annu. Rev. Ecol. Syst., 29(1): 543-66. https://doi. org/10.1146/annurev.ecolsys.29.1.543.

Otchere, F.A. 2019. A 50-year review on heavy metal pollution in the environment: Bivalves as bio-monitors. Afr. J. Environ. Sci. Tech., 13(6): $220-227$.

Otchere, F.A., C.R. Joiris and L. Holsbeek. 2003. Mercury in the bivalves Anadara (Senilia) senilis, Perna perna and Crassostrea tulipa from Ghana. Sci. Total Environ., 304: 369-375. https://doi.org/10.1016/S0048-9697(02)00582-X

Riget, F., P. Johansen and G. Asmund. 1996. Influence of length on element concentrations in blue mussels (Mytilus edulis). Mar. Pollut. Bull., 32(10): 74551. doi:10.1016/0025-326X(96)00067-7

Santana, V., G. Medina y A. Torre. 2014. El convenio de Minamata sobre el mercurio y su implementación en la región de América Latina y el Caribe. http:// www.mercuryconvention.org/Portals/11/documents/publications/informe_Minamata_LAC_ES_FINAL.pdf.

Scardino, A., R. De Nys, O. Ison, W. O’Connor and P. Steinberg. 2003. Microtopography and antifouling properties of the shell surface of the bivalve molluscs Mytilus galloprovincialis and Pinctada imbricata. Biofouling, 19: 221-230. doi: 10.1080/0892701021000057882.

Schudel, G., R.A. Miserendino, M.M. Veiga, P.C. Velásquez-López, P.S.J. Lees, S. Winland-Gaetz, J.R. Guimarães and B.A. Bergquist. 2018. An investigation of mercury sources in the Puyango-Tumbes River: using stable Hg isotopes to characterize transboundary Hg pollution. Chemosphere, 202: 777-787. https://doi.org/10.1016/j.chemosphere.2018.03.081.

Schudel, G., R. Kaplan, R.A. Miserendino, M.M. Veiga, P.C. Velásquez-López, J.R. Davée Guimarães and B.A. Bergquist. 2019. Mercury isotopic signatures of tailings from artisanal and small-scale gold mining (ASGM) in southwestern Ecuador. Sci. Total Environ., 686: 301-10. https:// doi.org/10.1016/j. scitotenv.2019.06.004.

Secretaria Nacional de Planificación y Desarrollo. 2009. Evaluación social y técnica de los recursos hídricos de las subcuencas de los ríos Jagua, Balao, Gala, Tengel y Siete, en la provincia de Azuay. https://es.scribd.com/doc/29929927/INVENTARIO-R-HIDRICOS-JAGUA-TENGUELGALA-SIETE. 
Sevillano, J.S., M. Cejudo-Gómez, A.M. Ramírez-Ojeda, F. Cámara-Martos and R. Moreno-Rojas. 2015. Risk profile of methylmercury in seafood. Curr. Opin. Food. Sci., 6: 53-60. https://doi.org/10.1016/j.cofs.2016.01.003.

Silva, F.S., W. Machado, F. Lisboa and D. Lacerda. 2003. Mercury accumulation in sediments of a mangrove ecosystem in SE Brazil. Water Air Soil Poll., 145: 67-77. https://doi.org/10.1023/A.

Tam, N. e Y. Wong. 1995. Spatial and temporal variations of heavy metal contamination in sediments of a mangrove swamp in Hong Kong. Mar. Pollut. Bull., 31(4-12): 254-261. https://doi.org/10.1016/0025-326X(95)00141-9.

Tarras-Wahlberg, N.H., A. Flachier, G. Fredriksson and S. Lane. 2000. Environmental impact of small-scale and artisanal gold mining in southern Ecuador. AMBIO, 29(8): 484-491. doi: 10.1579/0044-7447-29.8.484

U.S. Environmental Protection Agency. 1989. Risk assessment guidance for superfund. Vol. I Human Health Evaluation Manual (Part A). https://doi. org/ EPA/540/1-89/002.

U.S. Environmental Protection Agency. 2001. Water quality criterion for the protection of human health: methylmercury. Methylmercury water quality criterion. EPA-823-R-. https://doi.org/EPA-823-F-01-001.

U.S. Environmental Protection Agency. 2002. Calculating upper confidence limits for exposure point concentrations at hazardous waste sites. Washington.

Vane, C.H., I. Harrison, A.W. Kim, V. Moss-Hayes, B.P. Vickers and K. Hong. 2009. Organic and Metal contamination in surface mangrove sediments of south China. Mar. Pollut. Bull., 58: 134-144. https://doi.org/10.1016/j.marpolbul.2008.09.024.

Velásquez López, P.C., M.M. Veiga, B. Klein, J.A. Shandro and K. Hall. 2011. Cyanidation of mercury-rich tailings in artisanal and small-scale gold mining: identifying strategies to manage environmental risks in southern Ecuador. J. Clean. Prod., 19(9-10): 1125-1133. https://doi.org/10.1016/j. jclepro.2010.09.008.

World Health Organization. 1996. Guidelines for drinking water quality. Vol. 2. Geneva: WHO library.

World Health Organization. 2008. Guidance for identifying populations at risk from mercury exposure. Geneva. https://doi.org/10.1289/ehp.7856.

Zuykov, M., E. Pelletier, C. Belzile and S. Demers. 2011. Alteration of shell nacre micromorphology in blue mussel Mytilus edulis after exposure to freeionic silver and silver nanoparticles. Chemosphere, 84: 701-706. doi: 10.1016/j.chemosphere.2011.03.021

Zuykov, M., E. Pelletier, R. St-Louis, A. Checa and S. Demers. 2012. Biosorption of thorium on the external shell surface of bivalve mollusks: The role of shell surface microtopography. Chemosphere, 86: 680-683. https://doi.org/10.1016/j.chemosphere.2011.11.023

RECIBIDO/RECEIVED: 24/08/2019

APROBADO/APPROVED: 20/04/2020 